\title{
Numerical Simulations of Melt-Driven Double-Diffusive Fluxes in a Turbulent Boundary Layer beneath an Ice Shelf
}

\author{
Leo Middleton, ${ }^{\text {a,b }}$ Catherine A. VReugdenhil,,${ }^{\mathrm{a}, \mathrm{c}}$ Paul R. Holland, ${ }^{\mathrm{b}}$ and John R. Taylor ${ }^{\mathrm{a}}$ \\ ${ }^{a}$ Department of Applied Mathematics and Theoretical Physics, University of Cambridge, Cambridge, United Kingdom \\ ${ }^{\mathrm{b}}$ British Antarctic Survey, Cambridge, United Kingdom \\ ${ }^{\mathrm{c}}$ Department of Mechanical Engineering, University of Melbourne, Melbourne, Victoria, Australia
}

(Manuscript received 22 June 2020, in final form 18 September 2020)

\begin{abstract}
The transport of heat and salt through turbulent ice shelf-ocean boundary layers is a large source of uncertainty within ocean models of ice shelf cavities. This study uses small-scale, high-resolution, 3D numerical simulations to model an idealized boundary layer beneath a melting ice shelf to investigate the influence of ambient turbulence on doublediffusive convection (i.e., convection driven by the difference in diffusivities between salinity and temperature). Isotropic turbulence is forced throughout the simulations and the temperature and salinity are initialized with homogeneous values similar to observations. The initial temperature and the strength of forced turbulence are varied as controlling parameters within an oceanographically relevant parameter space. Two contrasting regimes are identified. In one regime doublediffusive convection dominates, and in the other convection is inhibited by the forced turbulence. The convective regime occurs for high temperatures and low turbulence levels, where it is long lived and affects the flow, melt rate, and melt pattern. A criterion for identifying convection in terms of the temperature and salinity profiles, and the turbulent dissipation rate, is proposed. This criterion may be applied to observations and theoretical models to quantify the effect of doublediffusive convection on ice shelf melt rates.
\end{abstract}

KEYWORDS: Ice shelves; Mixing; Turbulence

\section{Introduction}

Ice shelves are the floating extensions of ice sheets, found around Antarctica and Greenland. Regional ocean models of the cavities beneath them are often used to help predict the response of ice shelves to various oceanographic forcings (Holland et al. 2010). To calculate the response of the ice shelf to a given ocean state, the turbulent boundary layer in the upper tens of meters must be parameterized. The parameterization commonly used in ice shelf cavity models was developed based on observations under sea ice (McPhee et al. 1987), and then adapted for the under ice-shelf environment (Holland and Jenkins 1999). Observations necessary for parameterization validation were previously minimal. However, ice shelf borehole measurements have recently increased in quantity and quality (Davis and Nicholls 2019; Kimura et al. 2015; Begeman et al. 2018; Jenkins et al. 2010).

Davis and Nicholls (2019) analyzed turbulence measurements made beneath the Larsen C Ice Shelf. Temperatures within the cold-water cavity were measured as $-2.01^{\circ} \pm 0.05^{\circ} \mathrm{C}$ at about $2.6 \mathrm{~m}$ below the ice. Davis and Nicholls (2019) found their observations were consistent with the Holland and Jenkins (1999) parameterization, which assumes a sheardriven boundary layer, where stratification due to basal melting has a minimal effect.

The effects of stratification on turbulence within the ocean boundary layer beneath an ice shelf were examined using large-eddy simulations (LES) in Vreugdenhil and Taylor

Corresponding author: John Taylor, j.r.taylor@damtp.cam.ac.uk
(2019). They considered a steady flow past a dynamically melting boundary and found that, under strongly stratified conditions, shear-driven turbulence was reduced and even damped out. The parameterization of McPhee et al. (1987) is based on similar arguments and stratification acts to damp turbulence. Holland and Jenkins (1999) argued that stratification effects in the McPhee et al. (1987) parameterization have a minimal impact on cold cavity ice shelves, and so need not be included within regional ice cavity models. However, the work of Vreugdenhil and Taylor (2019) suggested that stratification effects may be important even for relatively cold far-field temperatures, especially if the shear turbulence is weak. Many ice shelf cavities, including those that are losing ice mass at the fastest rates (Rignot et al. 2013), are warm water cavities. Here the McPhee et al. (1987) parameterization, and LES (Vreugdenhil and Taylor 2019), suggest stratification plays a dominant role in the transport of heat and salt through the boundary layer.

Certain observations cannot be explained by the damping effect of stratification. Borehole observations made on the George VI Ice Shelf (Venables et al. 2014; Kimura et al. 2015), found layers (or "thermohaline staircases") in the temperature and salinity profiles adjacent to the ice. Although layers can form in fluids with a single stratifying component (Phillips 1972), Kimura et al. (2015) argued that the staircases observed beneath George VI Ice Shelf are associated with the difference between the molecular diffusivities of temperature and salinity. Thermohaline staircases can form when one scalar is unstably stratified while the other is stably stratified (Radko 2013), and here the melting ice provides a stable salinity profile and an unstable temperature profile. This configuration is 
called the "diffusive convection favorable" regime (when the unstable stratifying element is salinity, the regime is called "salt fingering favorable"). Staircases are a common signature of convection triggered by the difference in diffusivities; however, double-diffusive convection may occur without staircase formation. In double-diffusive convection, on average turbulence is generated through the release of potential energy, despite the density increasing with depth. The parameterization of McPhee et al. (1987) assumes the role of the diffusive buoyancy flux at a melting ice base is to create a stratification that damps turbulence, so the parameterization will not apply well if turbulent production is dominated by double-diffusive convection. The Kimura et al. (2015) hypothesis was that double-diffusive convection is forced at the ice base, leading to the signature staircases below, and we are primarily concerned with investigating this mechanism.

Kimura et al. (2015) compared the under-ice shelf regime to the laboratory experiment of Martin and Kauffman (1977). In this experiment a block of ice was floated atop a box of saltwater $\left(0^{\circ} \mathrm{C}\right.$ and $37.6 \mathrm{ppt}$ salinity). Convection was observed throughout the box that persisted for the length of the experiment ( 2 days). The diffusivity of heat is two orders of magnitude larger than the diffusivity of salt, so a diffusing thermal sublayer will thicken faster than a salt sublayer. In the Martin and Kauffman (1977) experiments the density was dominated by the cooled temperature profile beneath the salt boundary layer, causing a peak in density that triggered convection. The velocity field was not examined in these experiments, but a numerical study of a melting boundary was conducted by Keitzl et al. (2016), where similar convection was observed. The Keitzl et al. (2016) simulations showed convective plumes descending from a region immediately below the salt boundary layer, although here the far-field temperatures were larger, varying between $10^{\circ}$ and $24^{\circ} \mathrm{C}$. Martin and Kauffman (1977) did not observe staircase formation; however, their experimental setup had no ambient stratification. Turner (1968) observed the progressive formation of staircases when heating a stable salt stratification from below, suggesting staircases may form in a stable stratification when double-diffusive convection is forced by a destabilizing flux at the boundary. Kimura et al. (2015) argued that diffusive boundary fluxes as in Martin and Kauffman (1977) and a stable stratification as in Turner (1968) led to the staircases observed beneath George VI Ice Shelf. Following Martin and Kauffman (1977) we will not consider staircase formation, and instead we will seek to understand the response of ice-triggered convection to turbulent mixing.

The experiments and observations described above suggest that double diffusion is potentially important beneath ice shelves. However, it is not clear how double-diffusive convection will interact with turbulence occurring within an ice shelf-ocean boundary layer. In steady state it can be shown (using the three-equation model in section $2 \mathrm{~b}$ ) that doublediffusive convection implies a fresh salinity sublayer (below $\sim 4$ ppt), which was not observed by Kimura et al. (2015) or Martin and Kauffman (1977), implying the observed doublediffusive convection was transient. Nevertheless, the convection in the experiment of Martin and Kauffman (1977) was long lived, with a salinity sublayer growing on the diffusive time scale for salinity, thickening by $1 \mathrm{~cm}$ in around $20 \mathrm{~h}$. Gade (1979) noted that by agitating crushed ice within salty water, one could inhibit double-diffusive convection, which otherwise caused the meltwater to sink. Gade (1979) argued that convection was inhibited by the low-salinity boundary layer being mixed into the interior, where it had a dominant contribution to the density. The inhibition of double-diffusive convection by turbulence has also been observed in the ocean (Shibley and Timmermans 2019; Guthrie et al. 2013) and in laboratory experiments (Crapper 1976).

Although the observations from George IV Ice Shelf reported by Kimura et al. (2015) showed clear evidence for double-diffusive convection, Venables et al. (2014) noted that some turbulent shear profiles taken beneath George VI Ice Shelf showed low dissipation values concurrent with doublediffusive staircases, while others showed no staircases and high dissipation values. One hypothesis is that double-diffusive convection is suppressed when turbulence exceeds a critical threshold. Inspired by these observations, we will test this hypothesis and investigate double-diffusive convection forced by heat and salt fluxes at the ice boundary in the presence of ambient turbulence.

To investigate double-diffusion within an adjusting ice shelf-ocean boundary layer we use idealized, high-resolution numerical simulations, inspired by field observations. We force ambient turbulence to reach a target dissipation rate similar to measurements beneath George VI Ice Shelf (Venables et al. 2014), then consider the evolution of a dynamically melting boundary under a homogeneous initial condition for temperature and salinity. We vary the far-field temperature and forced dissipation rate across simulations as controlling parameters. The initial condition of uniform scalars is not designed to capture staircase formation, and the focus instead is the interaction between turbulence and double diffusion near the ice base.

Double-diffusive convection will be distinguished from "stratified turbulence" in this paper using the turbulent vertical buoyancy flux, defined as $\left\langle w^{\prime} b^{\prime}\right\rangle$, i.e., the correlation between the fluctuating vertical velocity $w^{\prime}$, where angle brackets denote a horizontal average and primes are departures from this average, and the fluctuating buoyancy $b=g \alpha T^{\prime}-g \beta S^{\prime}$ for $g$ the gravitational acceleration, $T$ the temperature, $S$ the salinity and $(\alpha, \beta)$ constant coefficients of thermal expansion and haline contraction, respectively. The buoyancy flux determines the energetic contribution of the buoyancy field (through potential energy) to the turbulent kinetic energy (TKE) as described in section 2c. Negative values imply that the buoyancy flux acts as a sink of TKE, and positive values imply the buoyancy flux increases the TKE. If double-diffusive convection is the dominant mechanism we expect a positive buoyancy flux $\left(\left\langle w^{\prime} b^{\prime}\right\rangle>0\right)$, otherwise stratification will dampen turbulence on average $\left(\left\langle w^{\prime} b^{\prime}\right\rangle<0\right)$. However, turbulent buoyancy flux is a noisy measure of energy exchange that may locally change sign, as it depends on advection. This motivates dividing potential energy into two parts; an "available" potential energy (APE), that exchanges energy back and forth with the TKE via $\left\langle w^{\prime} b^{\prime}\right\rangle$ (advection); and a "background" potential energy (BPE) that exchanges energy with the APE based on 
the mixing of the buoyancy field (diffusion) (Winters et al. 1995).

For single component fluids the diffusive energy exchange between APE and BPE is one way, i.e., mixing always acts to increase BPE (Winters et al. 1995). The distinction between APE and BPE is complicated for double-diffusive fluids, as buoyancy gradients can sharpen due to diffusion (Merryfield 2000). However, Middleton and Taylor (2020) applied the APE/BPE framework to double-diffusion, where now diffusion can cause "un-mixing" i.e., a release of BPE into APE. Middleton and Taylor (2020) obtained a simplified criterion to identify transfers of energy from BPE to APE (section 4a), and here we will apply this criterion to quantify the importance of double diffusion in our simulations.

Section 2 outlines our simulation set up, focusing on the simulation geometry, forcing and numerical details of the grid and its relation to the turbulent length scales. In section 3 we discuss the simulation evolution, considering differences between convective and stable regimes. Then in section 4 we consider the diapycnal flux in our simulations. First, we give a review of the criterion introduced by Middleton and Taylor (2020), then we show that convection in our simulations is caused by a region of negative diapycnal buoyancy flux near the ice base and it is well described using the framework from Middleton and Taylor (2020). The region is also well described by the density ratio and buoyancy Reynolds number, which we formulate into a criterion for the inhibition of double-diffusive convection by externally forced turbulence. Finally, in section 5 we apply our criterion to the diffusive solution, providing a point of comparison between our simulations, those of Vreugdenhil and Taylor (2019) and the observations. Concluding remarks are offered in section 6 .

\section{Methods}

The ocean boundary layer beneath a melting ice shelf is simulated in a rectangular box domain (Fig. 1). We use periodic boundary conditions in the horizontal $x, y$ directions and impenetrable conditions in the vertical $z$ direction. Dynamic melting boundary conditions are imposed on the temperature and salinity fields locally across the top surface of the domain, along with a no-slip velocity condition. A no-flux, free slip condition is applied at the base. The simulations are initialized with a homogeneous temperature and salinity, which are restored to initial values below an "observation" region of 2.6-m depth. Isotropic turbulence is forced at length scales larger than the observation region, using a methodology taken from Wang et al. (1996), applied as a forcing term discussed in section $2 \mathrm{a}$. The mechanical forcing is designed to achieve a prescribed rate of turbulent kinetic energy dissipation, with values chosen similar to George VI Ice Shelf observations. The mechanically forced turbulence is intended to represent processes missing from the simulations, such as shear-driven turbulence, internal wave breaking, or interior double-diffusive convection.

\section{a. Governing equations}

Our simulations solve the incompressible, nonhydrostatic, Boussinesq Navier-Stokes equations, with terms to apply

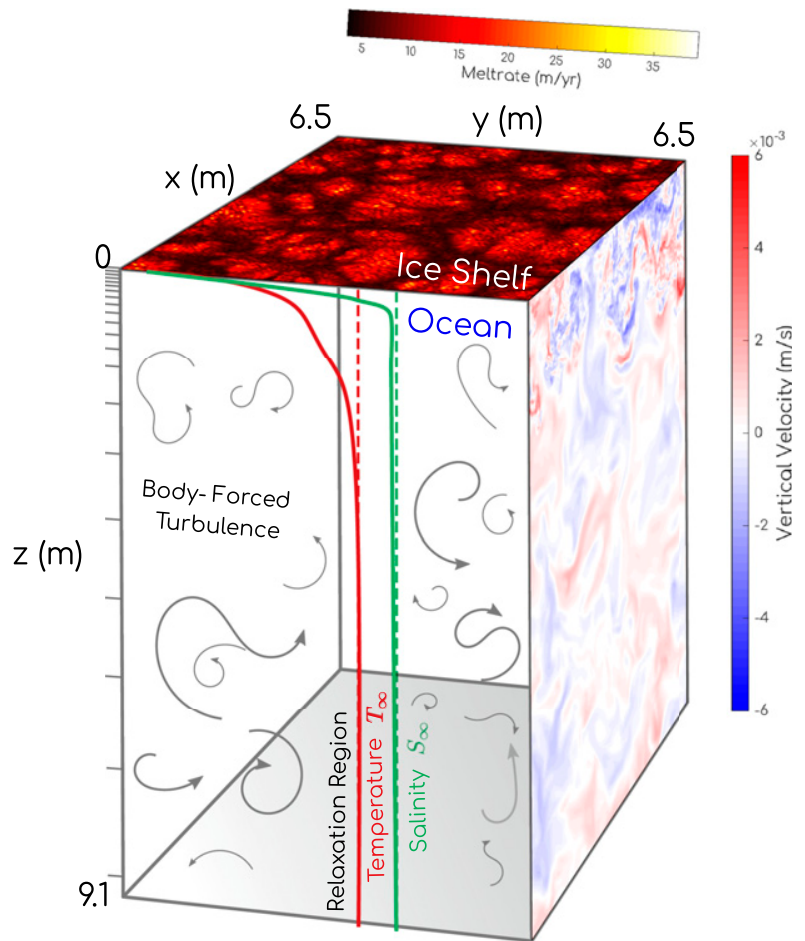

FIG. 1. Schematic of model domain with included snapshots of vertical velocity field and melt rate for warm, low mechanical forcing simulation 1B listed in Table 1.

mechanical velocity forcing and far-field scalar relaxation. These equations are

$$
\begin{aligned}
& \frac{D \mathbf{u}}{D t}=-\frac{1}{\rho_{0}} \nabla p+\nu \nabla^{2} \mathbf{u}-\frac{\Delta \rho}{\rho_{0}} g \mathbf{k}+\underbrace{\frac{\varepsilon_{0} \mathbf{u}}{\langle\mathbf{u} \cdot \mathbf{u}\rangle}}_{\text {Mechanical forcing }}, \\
& \nabla \cdot \mathbf{u}=0, \\
& \frac{D T}{D t}=\kappa_{T} \nabla^{2} T-\underbrace{\frac{1}{\tau_{0}}\left(\langle T\rangle-T_{\infty}\right) r(z)}_{\text {Far-field relaxation }}, \\
& \frac{D S}{D t}=\kappa_{S} \nabla^{2} S-\overbrace{\frac{1}{\tau_{0}}\left(\langle S\rangle-S_{\infty}\right) r(z)}, \\
& \frac{\Delta \rho}{\rho_{0}}=-\alpha\left(T-T_{0}\right)+\beta\left(S-S_{0}\right),
\end{aligned}
$$

where $D / D t=\partial / \partial t+\mathbf{u} \cdot \nabla$ is the material derivative for $\mathbf{u}=(u$, $v, w)$ the $3 \mathrm{D}$ velocity field with respect to position vector $\mathbf{x}=$ $(x, y, z)$ and $p$ pressure. The density is $\rho$, with $\rho_{0}=1000 \mathrm{~kg} \mathrm{~m}^{-3}$ the reference density and $\Delta \rho=\rho-\rho_{0}$. We use $\nu=1.8 \times$ $10^{-6} \mathrm{~m}^{2} \mathrm{~s}^{-1}$ as kinematic viscosity, and $g=9.81 \mathrm{~m} \mathrm{~s}^{-2}$ as gravitational acceleration. The term $T$ is the temperature field $\left({ }^{\circ} \mathrm{C}\right)$, with $T_{0}$ and $T_{\infty}$ the reference and far-field temperatures, respectively. Likewise, $S$ is the salinity field in parts per thousand, with $S_{0}$ and $S_{\infty}$ the reference and far-field salinities. The molecular diffusivities are $\kappa_{T}=1.3 \times 10^{-7} \mathrm{~m}^{2} \mathrm{~s}^{-1}$ for temperature and $\kappa_{S}=7.4 \times 10^{-10} \mathrm{~m}^{2} \mathrm{~s}^{-1}$ for salt. Finally, the 
TABLE 1. Simulation parameters. Values for $\Delta T, \Delta S$, and $R_{\rho}$ are averaged across the simulation. The turbulent vertical buoyancy flux $\left\langle w^{\prime} b^{\prime}\right\rangle$ is averaged across the simulation for depths $0.1 \mathrm{~m}<z<2.6 \mathrm{~m}$ for the first $35 \mathrm{~h}$ to enable comparison across simulations run for different lengths of time. Simulations $1 \mathrm{~A}-2 \mathrm{~B}$ are convecting throughout the simulated time, and simulations $2 \mathrm{C}-6$ are not.

\begin{tabular}{lllcccc}
\hline \hline Mean forced dissipation & \multicolumn{3}{c}{} & \multicolumn{2}{c}{ Turbulent buoyancy flux } \\
$\varepsilon_{0}\left(\mathrm{~m}^{2} \mathrm{~s}^{-3}\right)$ & \multicolumn{1}{c}{$T_{\infty}\left({ }^{\circ} \mathrm{C}\right)$} & $\Delta T\left({ }^{\circ} \mathrm{C}\right)$ & $\Delta S(\mathrm{ppt})$ & $\begin{array}{c}\text { Density ratio } \\
R_{\rho}=\beta \Delta S / \alpha \Delta T\end{array}$ \\
\hline 1A & No forcing & 0.15 & 1.49 & 14.4 & $3.5 \times 10^{-9}$ & 197 \\
1B & $(8.7 \pm 1.8) \times 10^{-11}$ & 0.15 & 1.43 & 15.4 & $2.3 \times 10^{-9}$ & $2.2 \times 10^{-9}$ \\
1C & $(1.7 \pm 0.15) \times 10^{-9}$ & 0.15 & 1.43 & 15.5 & $6.0 \times 10^{-12}$ & 220 \\
2A & No forcing & -2.15 & $5.8 \times 10^{-3}$ & $9.64 \times 10^{-2}$ & $5.1 \times 10^{-12}$ & 338 \\
2B & $(8.7 \pm 1.8) \times 10^{-11}$ & -2.15 & $5.4 \times 10^{-3}$ & $10.3 \times 10^{-2}$ & $-3.4 \times 10^{-11}$ & 383 \\
2C & $(1.7 \pm 0.15) \times 10^{-9}$ & -2.15 & $5.9 \times 10^{-3}$ & $9.46 \times 10^{-2}$ & $-5.2 \times 10^{-14}$ & 325 \\
3 & $(8.7 \pm 1.7) \times 10^{-11}$ & -2.16 & $7.3 \times 10^{-4}$ & $1.04 \times 10^{-2}$ & $-5.4 \times 10^{-13}$ & 293 \\
4 & $(8.7 \pm 1.7) \times 10^{-11}$ & -2.161 & $1.8 \times 10^{-4}$ & $2.47 \times 10^{-3}$ & $-8.7 \times 10^{-14}$ & 274 \\
5 & $(8.7 \pm 1.7) \times 10^{-11}$ & -2.1613 & $1.5 \times 10^{-5}$ & $1.81 \times 10^{-3}$ & $-1.4 \times 10^{-15}$ & 242 \\
6 & $(8.7 \pm 1.7) \times 10^{-11}$ & -2.161325 & $4 \times 10^{-7}$ & $4.00 \times 10^{-6}$ & & 220 \\
\hline
\end{tabular}

constants $(\alpha, \beta)=\left(3.87 \times 10^{-5}{ }^{\circ} \mathrm{C}^{-1}, 7.86 \times 10^{-4} \mathrm{ppt}^{-1}\right)$ (Jenkins et al. 2010) are the coefficients of thermal expansion and haline contraction. Within the relaxation term, the angled brackets $\langle\cdot\rangle$ represent a horizontal average and $\tau_{0}=200 \mathrm{~s}$ is the relaxation time scale, chosen based on a far-field velocity scale of $\sim 5 \mathrm{~cm} \mathrm{~s}^{-1}$ and a domain height $\sim 10 \mathrm{~m}$. The term $r(z)=$ $0.5[\tanh (10-2 z)+1]$ ensures that the relaxation term only acts in the far-field and $r \simeq 10^{-4}$ at $z=2.6 \mathrm{~m}$. Therefore, the temperature and salinity are not forced at a depth similar to the mooring measurements made beneath the ice at Larsen $\mathrm{C}$ Ice Shelf and George VI Ice Shelf. Our simulations do not include the effect of Earth's rotation since the nonrelaxed part of our domain is small $(2.6 \mathrm{~m})$ and there is no mean flow, so rotational effects will be weak. We explain the mechanical forcing term in section 2c.

\section{b. Simulations details}

Equations (1)-(5) are discretized using a pseudospectral method in the horizontal, and a second-order finite difference scheme in the vertical (see Taylor 2008). The 2/3 dealiasing technique (Orszag 1971) is applied whereby the Fourier coefficients associated with the largest $1 / 3$ of wavenumbers are set to zero. This has the effect of dissipating scalar variance on scales smaller than $3 \Delta$, where $\Delta$ is the horizontal grid spacing. In regions of the flow where the simulations do not resolve the Batchelor scale, the dealiasing procedure and the numerical dissipation associated with the finite difference scheme acts like an implicit subgrid-scale model by removing small-scale variance. An implicit Crank-Nicholson method is used to time step the viscous and diffusive terms, and a third-order RungeKutta method for other terms.

Full details on the melt condition can be found in Vreugdenhil and Taylor (2019); however, in summary, the method solves the diffusive three-equation model (Frank 1950) at the boundary, i.e., the equations

$$
\begin{aligned}
\rho_{i} L_{i} m & =\left.c_{p} \rho_{w} \kappa_{T} \frac{\partial T}{\partial z}\right|_{b}, \\
\rho_{i} S_{b} m & =\left.\rho_{w} \kappa_{S} \frac{\partial S}{\partial z}\right|_{b}, \\
T_{b} & =\lambda_{1} S_{b}+\lambda_{2}+\lambda_{3} P,
\end{aligned}
$$

where $m$ is the melt rate, $T_{b}$ is the temperature at the ice base, and $S_{b}$ is the salinity at the ice base. The constants are $c_{p}=$ $3974 \mathrm{~m}^{2} \mathrm{~s}^{-2} \mathrm{~kg}^{-1}{ }^{\circ} \mathrm{C}^{-1}$ for specific heat capacity, $L_{i}=3.35 \times$ $10^{5} \mathrm{~m}^{2} \mathrm{~s}^{-2} \mathrm{~kg}^{-1}$ for latent heat of fusion and $\rho_{w}=1000 \mathrm{~kg} \mathrm{~m}^{-3}$, $\rho_{i}=920 \mathrm{~kg} \mathrm{~m}^{-3}$ for the densities of seawater and ice, respectively. Here $\lambda_{1}=-5.73 \times 10^{-2}{ }^{\circ} \mathrm{C}, \lambda_{2}=8.72 \times 10^{-2}{ }^{\circ} \mathrm{C}$ and $\lambda_{3}=-7.53 \times 10^{-4}{ }^{\circ} \mathrm{C} \mathrm{dbar}^{-1}$ (Jenkins et al. 2010). The gradients $\partial S / \partial z$ and $\partial T / \partial z$ are calculated at the boundary, at each time step, in each grid cell, to give a dynamic melt condition. The heat and salt flux through the ice is set to zero, as suggested by Holland and Jenkins (1999). We neglect the volume input of meltwater, as the interface moves slowly compared to the turbulent velocities.

Resolving the diffusive length scales for salinity everywhere in the domain is prohibitively expensive as the molecular diffusivity is small. Previous numerical simulations (Gayen et al. 2016) used artificially large diffusivities to resolve doublediffusive behavior. However, this may lead to underestimation of the double-diffusive effects. We use realistic molecular diffusivities for temperature and salinity and use a fine grid spacing to resolve the smallest diffusive scales within the scalar sublayers. In the turbulent region beneath these sublayers, turbulent fluxes will dominate heat and salt transport, and so it is sufficient that our simulations resolve the smallest velocity scales within the observation region, $z<2.6 \mathrm{~m}$, using typical resolution criteria. In other words, the simulations can be classified as direct numerical simulations (DNS) near the ice where they resolve the scalar and velocity gradients and implicit large-eddy simulations farther from the ice where they resolve the turbulent eddies but not all scales of tracer variance. A similar approach has been used before to simulate turbulent scalar transport of active tracers (e.g., Hickel et al. 2007; Scalo et al. 2012). For further details relating to the grid spacing see the appendix.

Table 1 lists the simulation runs. These are split into "warm" simulations $\left(0.15^{\circ} \mathrm{C}\right)$, with temperatures similar to George VI Ice Shelf (Venables et al. 2014), and "cold" simulations $\left(-2.15^{\circ} \mathrm{C}\right)$ similar to cold-water ice shelves such as Larsen C Ice Shelf (Davis and Nicholls 2019). The warm temperatures are similar to the Martin and Kauffman (1977) experiment. We also consider small far-field temperatures (simulations 3-6) to investigate the simulation evolution when stratification is weak. The initial salinity $S_{\infty}=34.572 \mathrm{ppt}$, the same across simulations, is taken from an 
average of CTD profiles at 2.6-m depth from George VI Ice Shelf. The temperature and salinity fields are initialized with constant values $T_{\infty}$ and $S_{\infty}$ in each simulation. We consider two values of the target dissipation rate $\varepsilon_{0}$ as described below in section 2c.

\section{c. Mechanical forcing}

The mechanical forcing term labeled in Eq. (1) is formulated so, in the absence of convection and buoyancy effects, the mean turbulent dissipation rate will be approximately $\varepsilon_{0}$. The volume-averaged turbulent kinetic energy (TKE) budget is

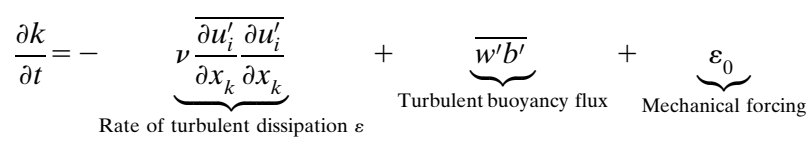

where $k=(1 / 2)\left[\overline{\left(u^{\prime}\right)^{2}}+\overline{\left(v^{\prime}\right)^{2}}+\overline{\left(w^{\prime}\right)^{2}}\right]$ is the TKE, an overbar denotes a volume average, and primes are departures from the volume average. There is an implicit sum over repeated subscripts.

For quasi-steady states, the rate of change of TKE is small. If the buoyancy flux is also small, the dominant energy balance is $\varepsilon \simeq \varepsilon_{0}$. We will refer to $\varepsilon_{0}$ as the target dissipation rate. In practice, target values of $1 \times 10^{-10}$ and $2 \times 10^{-9} \mathrm{~m}^{2} \mathrm{~s}^{-3}$ resulted in dissipation rates of $(8.7 \pm 1.8) \times 10^{-11} \mathrm{~m}^{2} \mathrm{~s}^{-3}$ and $(1.7 \pm 0.15) \times 10^{-9} \mathrm{~m}^{2} \mathrm{~s}^{-3}$ within the passive spinup simulation at steady state. The target value of $1 \times 10^{-10} \mathrm{~m}^{2} \mathrm{~s}^{-3}$ is similar to the lower values measured beneath George VI Ice Shelf (Venables et al. 2014) and the forced value of $2 \times 10^{-9} \mathrm{~m}^{2} \mathrm{~s}^{-3}$ is similar to the lower values measured beneath the Larsen $\mathrm{C}$ Ice Shelf (Davis and Nicholls 2019).

The turbulent buoyancy flux $\overline{w^{\prime} b^{\prime}}$ represents energy transfer between kinetic and potential energy. In steady state, the melt condition and relaxation provide sources of potential energy. When the buoyancy flux is included, the dissipation rate will increase or decrease relative to the equilibrium rate $\varepsilon_{0}$ depending on the sign of $\overline{w^{\prime} b^{\prime}}$.

As in Wang et al. (1996), we only force the lowest wavenumbers and allow the turbulent cascade to form naturally at higher wavenumbers. This method of forcing stratified turbulence has been used extensively by previous authors (Rao and de Bruyn Kops 2011; Taylor and Stocker 2012) to simulate stratified turbulence, including by Taylor et al. (2019) to test the assumptions underlying studies of ocean mixing. Specifically, we force wavelengths greater than $L / 2.5$ and less than $L$, where $L$ is the domain size, following Wang et al. (1996). We want the smallest forced wavelength (i.e., $L / 2.5$ ) to be no smaller than the height of the observation region $(2.6 \mathrm{~m})$, which sets the minimum vertical length scale as $L=2.5 \times 2.6 \mathrm{~m}=6.5 \mathrm{~m}$. In the relaxation region we set both the domain width and height equal to the minimum scale of $6.5 \mathrm{~m}$ to achieve an isotropic forcing.

\section{Results}

\section{a. Flow regimes}

In the case with no forced turbulence, once the scalars are initialized, the sublayers in temperature and salinity begin to grow. The thermal sublayer grows faster than the haline sublayer due to the larger molecular diffusivity of temperature. This leads to a double-diffusive boundary layer structure, comparable to the lower half of a double-diffusive interface (e.g., Carpenter et al. 2012), with a stable "core" where the salinity dominates the density above a "diffusive boundary layer" where the temperature dominates the density and leads to a peak in density. This behavior is reproduced by the diffusive solution for $T / S$ evolution beneath a melting interface from Martin and Kauffman (1977). The peak in density may then become unstable leading to diffusive convection. Figure 2 shows profiles of horizontally averaged scalar fields for the cold, low mechanical forcing case $2 \mathrm{~B}$ at various times. The early time behavior of this simulation is similar to the unforced case and matches the diffusive solution. The plots are magnified to show the peak in mean density; however, this variation is a small proportion of the total density difference which is largely contained in the $T$ and $S$ sublayers, as shown in the inset.

The addition of forced turbulence enhances vertical mixing of temperature and salinity, which acts to remove the middepth density maximum. As the flow evolves, the density peak increases in depth and decreases in magnitude. Changes in the magnitude of the density peak are sometimes dominated by salinity and sometimes by temperature, and hence cannot be attributed to mixing of one scalar alone. In the warmer simulations $1 \mathrm{~B}, 1 \mathrm{C}$ and the unforced simulations $1 \mathrm{~A}, 2 \mathrm{~A}$, the decrease in density peak magnitude is slow and the peak persists throughout the simulations $(50+\mathrm{h}$ in all cases). This suggests that if the conditions are sufficient to trigger convection, it may be long lasting. In the cold, mechanically forced cases $(2 \mathrm{~B}, 2 \mathrm{C}$, $3,4,5,6)$ the mean density profiles do become gravitationally stable during the simulation, with differing transition times dependent on the thermal and mechanical forcing. Case 2B, shown in Fig. 2, took the longest of the cold cases to transition; the density profile has a peak in the mean profile until around $200 \mathrm{~h}$, although at $100 \mathrm{~h}$ the peak is not visible without greater magnification. However, the lack of a peak in the mean density profile does not imply that no double-diffusive convection is present. It is possible that the stratification is still adding energy to the TKE via an upgradient turbulent vertical buoyancy flux as discussed below in section 4 a. Therefore, we use the sign of $\left\langle w^{\prime} b^{\prime}\right\rangle$ to identify double-diffusive convection.

Figure 3 shows the horizontally averaged turbulent vertical buoyancy flux $\left\langle w^{\prime} b^{\prime}\right\rangle$ for three simulations: the warm, low mechanical forcing case $1 \mathrm{~A}$; the cold, low mechanical forcing case $2 \mathrm{~B}$ and the cold, high mechanical forcing case $2 \mathrm{C}$. Positive values of $\left\langle w^{\prime} b^{\prime}\right\rangle$ indicate that the potential energy is acting as a source of TKE, and negative values indicate that TKE is converted into potential energy. Initially, a region with $\left\langle w^{\prime} b^{\prime}\right\rangle>0$ descends through the domain in all cases, due to the density peak discussed above. In the cold, low mechanical forcing cases, there are areas of $\left\langle w^{\prime} b^{\prime}\right\rangle<0$ visible after some time. In case $2 \mathrm{~B}$ these patches are initially confined near the ice base, but at later times they descend throughout the domain. In case $2 \mathrm{C}$ the regions quickly develop throughout the domain; however, regions of $\left\langle w^{\prime} b^{\prime}\right\rangle$ are still present, despite not being the dominant contribution to the horizontal average. In the warm case $1 \mathrm{~B}$, $\left\langle w^{\prime} b^{\prime}\right\rangle>0$ throughout the simulation length $(50 \mathrm{~h})$, and there are no regions of $\left\langle w^{\prime} b^{\prime}\right\rangle<0$ descending through the domain. 
(a)

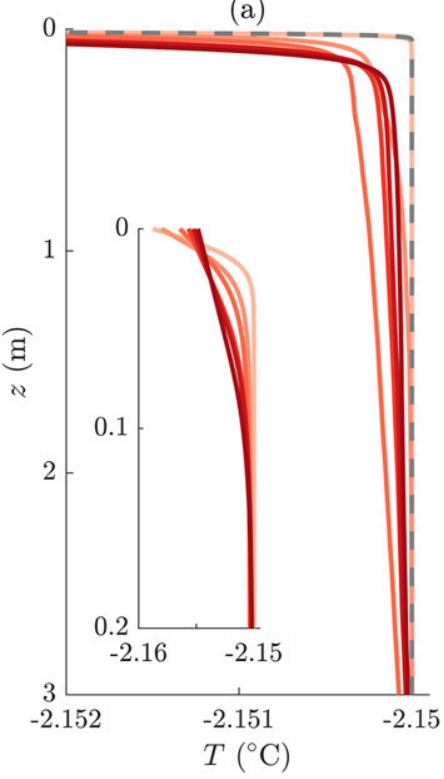

(b)

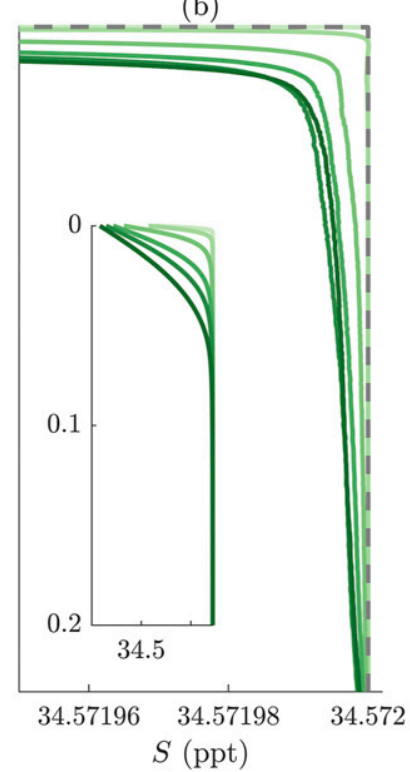

(c)

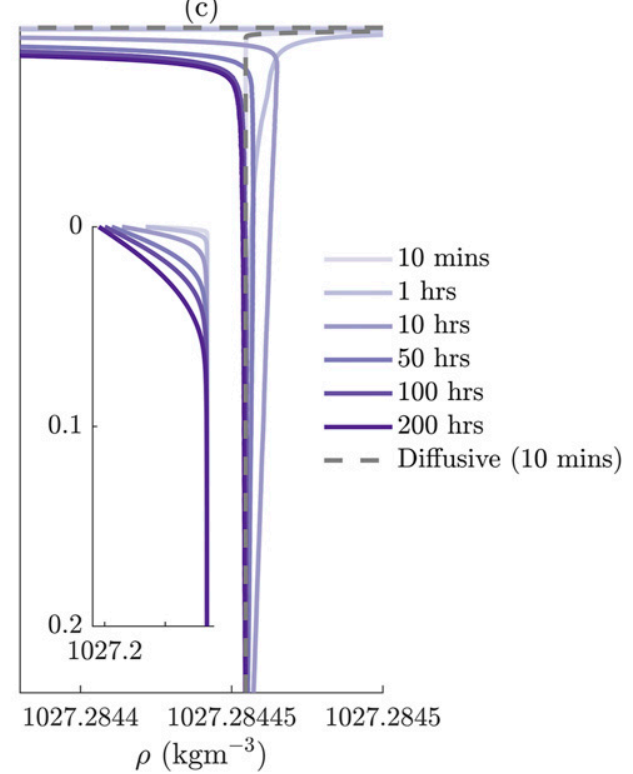

FIG. 2. Horizontally averaged (a) temperature, (b) salinity, and (c) density averaged over an hour, at hours 1, 10, 50, 100, and 200 for the cold, low mechanical forcing simulation 2B. Additionally we have shown the diffusive solution profiles (Martin and Kauffman 1977) and the simulation profiles at $t=10 \mathrm{~min}$, which compare well. The inset shows the upper $20 \mathrm{~cm}$ in each panel, showing the full variation in the scalars.

The mean density profile is relatively effective at determining the sign of $\left\langle w^{\prime} b^{\prime}\right\rangle$. In case $2 \mathrm{C}$ the density profile transitions after $\sim 4 \mathrm{~h}$, close to the time when $\left\langle w^{\prime} b^{\prime}\right\rangle$ changes sign $(\sim 2 \mathrm{~h})$. However, there are still regions of $\left\langle w^{\prime} b^{\prime}\right\rangle>0$ in case $2 \mathrm{C}$, and in case $2 \mathrm{~B}$ the changing sign of $\left\langle w^{\prime} b^{\prime}\right\rangle$ is sufficiently noisy that the direction of energy transfer between APE and TKE is not clear. Some variation in the sign of $\left\langle w^{\prime} b^{\prime}\right\rangle$ may be attributed to reversible exchanges between potential energy and TKE i.e., "stirring" (Winters et al. 1995); however, double-diffusive effects may also be responsible.
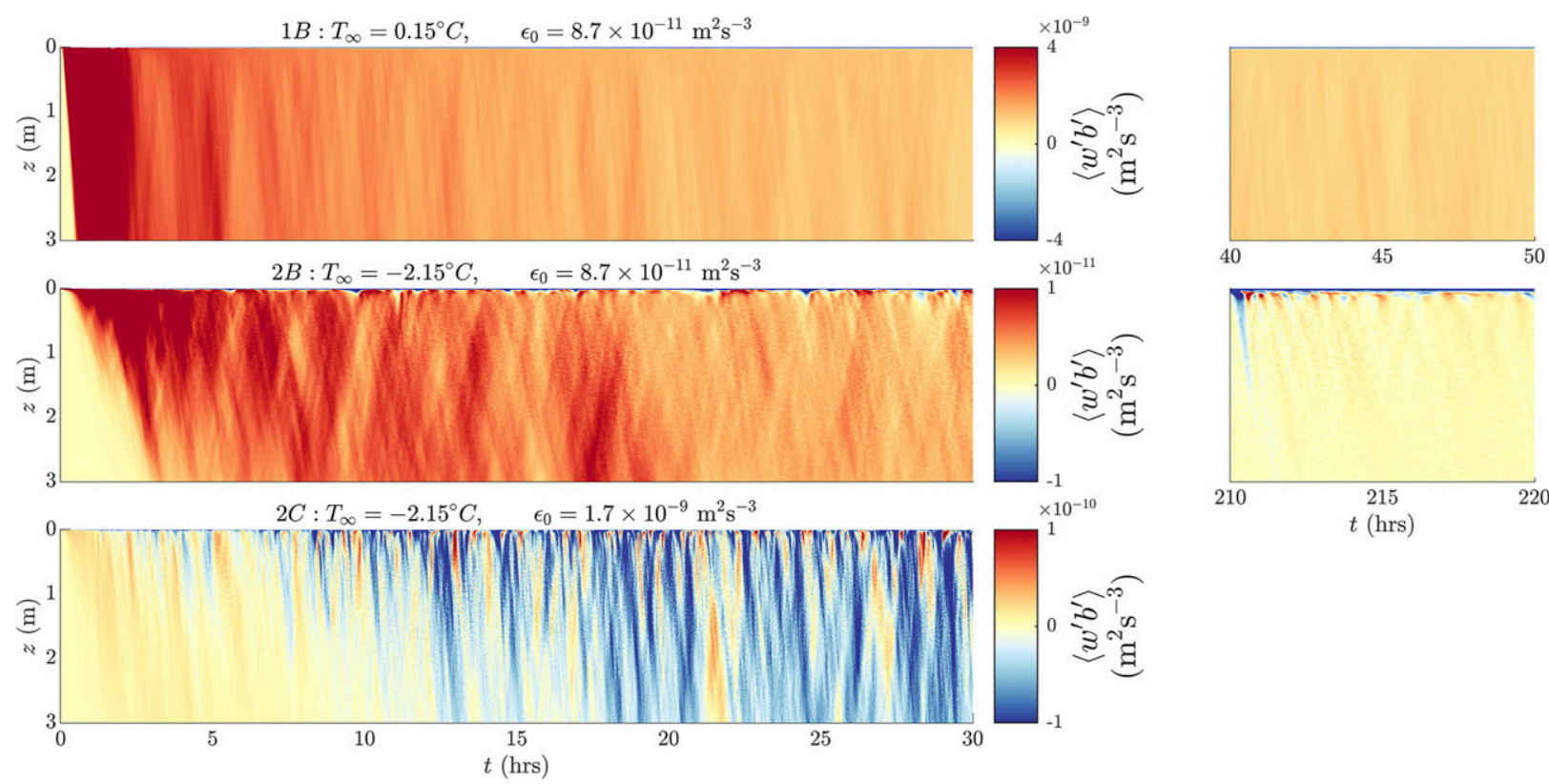

FIG. 3. Time evolution of the horizontally averaged turbulent buoyancy flux $\left\langle w^{\prime} b^{\prime}\right\rangle$ for simulation runs $2 \mathrm{~B}$ (cold, low mechanical forcing), 2C (cold, high mechanical forcing), and 1B (warm, high mechanical forcing). Positive values signify stratification acting to transfer available potential energy to turbulent kinetic energy and negative values indicate stratification acting to transfer turbulent kinetic energy into available potential energy. The first $30 \mathrm{~h}$ of each simulation are shown concurrently, and then later times are shown for simulations $1 \mathrm{~A}$ and $2 \mathrm{~B}$. 

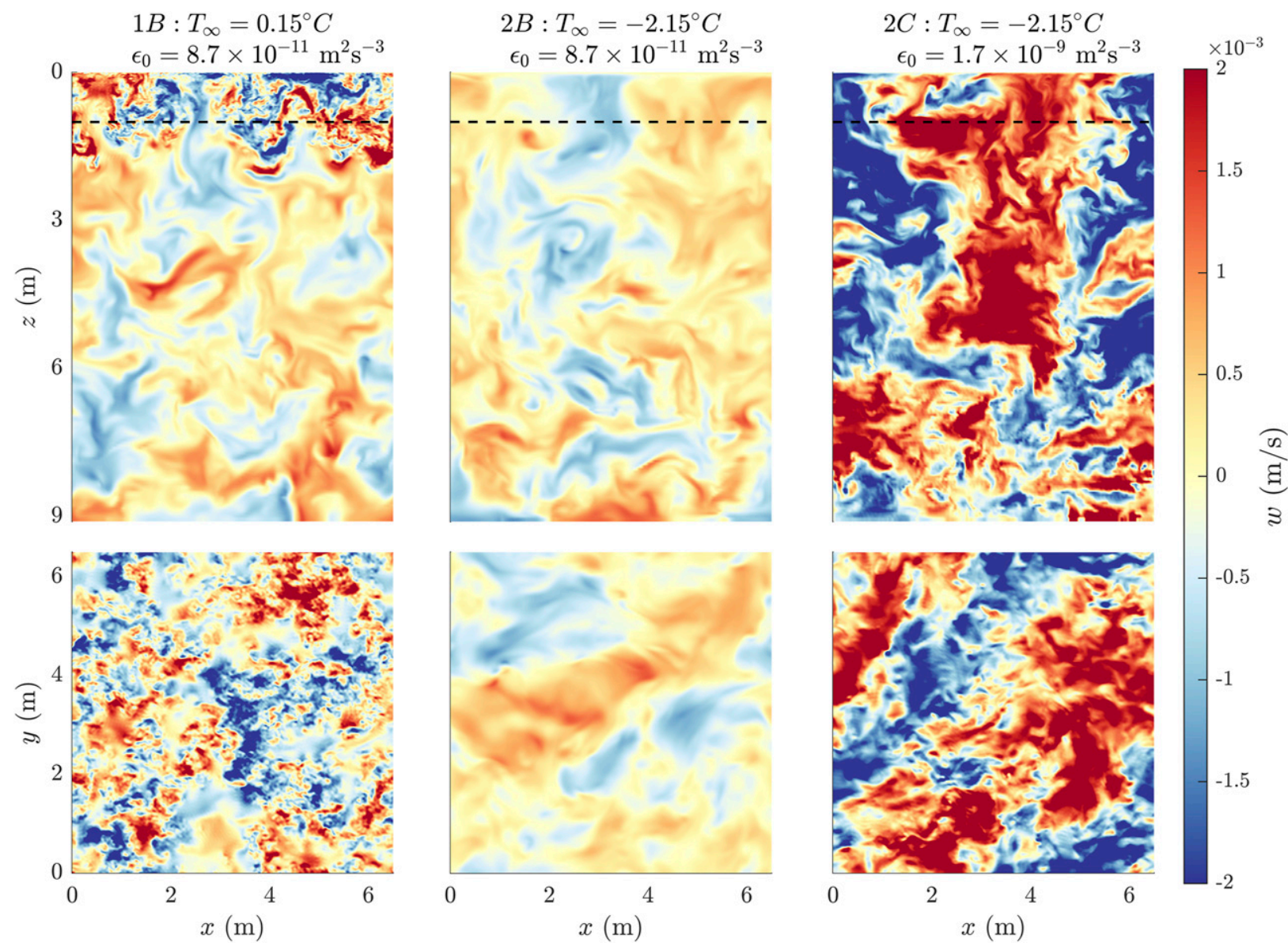

FIG. 4. Vertical velocity slices at $t=1 \mathrm{~h}$ for simulation runs 2B (cold, low mechanical forcing), 2C (cold, high mechanical forcing), and 1B (warm, high mechanical forcing). All plots are on the same color scale to illustrate relative magnitudes of vertical velocities. Horizontal slices (lower panels) taken at 1-m depth (location shown with dotted line in upper panels).

The magnitude of $\left\langle w^{\prime} b^{\prime}\right\rangle$ is also relevant. When $\left\langle w^{\prime} b^{\prime}\right\rangle$ is large and positive, it can dominate the TKE budget, but for small values it may not be energetically important. Figure 4 shows a snapshot of the vertical velocity field $w$ for the three simulations $1 \mathrm{~B}, 2 \mathrm{~B}$, and $2 \mathrm{C}$ at $t \sim 2 \mathrm{~h}$. The influence of the descending region of elevated buoyancy flux (see Fig. 3) is visible in Fig. 4 in case $1 \mathrm{~B}$ as an elevated value of $w$ close to the ice base that moves down through the domain. However, in the cold cases $2 \mathrm{~B}$ and $2 \mathrm{C}$ there is no visible contrast in the vertical velocity field. Even when buoyancy flux does not affect the velocity field, preferential diffusion may still affect the evolution of the scalar profiles that determine the melt rate.

In our convecting simulations, the magnitude of $\left\langle w^{\prime} b^{\prime}\right\rangle$ can be approximated by $g \alpha\left\langle w^{\prime} T^{\prime}\right\rangle$ away from the ice, which in turn can be approximated using the melt rate (not shown). The dominant mode of scalar transport is the large-scale forced eddies, amplified by the convective motions as shown in the warm case in Fig. 4. We show that the largest scales are responsible for the majority of the scalar fluxes in Fig. 5 by considering the fluxes in wavenumber space. The cospectrum of the scalar flux is calculated as $\langle\hat{w} \hat{\Theta} *\rangle$ for scalars $\Theta$, with . denoting the Fourier transform and $*$ denoting the complex conjugate. We show the turbulent scalar fluxes for $\Theta=b^{\prime}, g \alpha T^{\prime}$, $g \beta S^{\prime}$, i.e., the turbulent buoyancy flux, and the thermal and haline components of the turbulent buoyancy flux. The scalar fluxes are averaged between 1 and $3 \mathrm{~m}$ away from the ice, and then integrated between 0 and $k$, the radial wavenumber. The convergence of the integral in Fig. 5 shows that the largest scales are responsible for the majority of the integrated turbulent scalar fluxes, which suggests the details of the smallest scales (which we do not resolve) will have a small effect on the scalar fluxes. We have marked the cutoff frequency $k_{c}$ in the application of the $2 / 3$ dealiasing rule (see section $2 b$ ). The thermal component of the buoyancy flux dominates the buoyancy flux in Fig. 5, which holds throughout the convective regime.

\section{b. Melt}

Figure 6 shows the horizontally averaged melt rate as a function of time. The diffusive theory is accurate for early times in all cases. After the diffusive phase, all simulations show an increase in the melt rate due to turbulent mixing. For the cases with persistent convection (i.e., all apart from the cold, high mechanical forcing case 2C), the melt rate continues to decrease as $t^{-1 / 2}$ after the onset of convection. This can be 

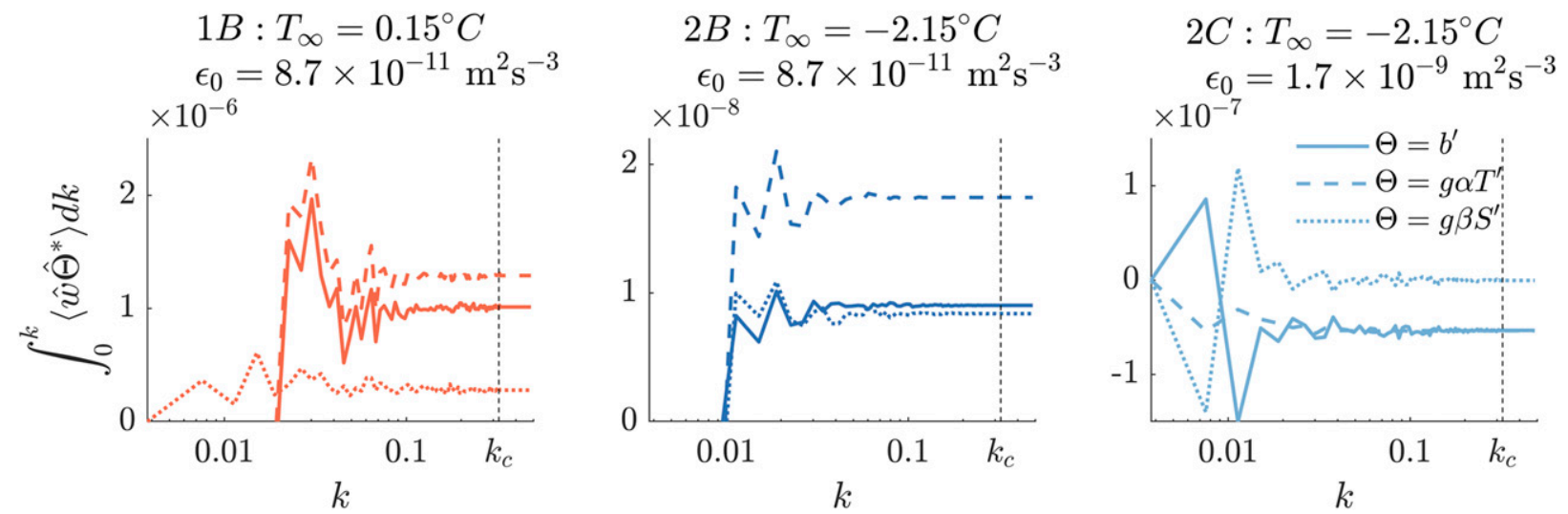

FIG. 5. Turbulent flux of temperature, salinity, and buoyancy, averaged between 1- and 3-m depth, integrated in Fourier space up to wavenumber $k$. Values taken from 3D fields at $t=1 \mathrm{~h}$ for simulation runs 2B (cold, low mechanical forcing), 2C (cold, high mechanical forcing), and 1B (warm, high mechanical forcing) as in Fig. 4. The Fourier transform is denoted using $\hat{.}$, and the complex conjugate is denoted by *. The wavenumber $k=\sqrt{k_{x}^{2}+k_{y}^{2}}$ is the horizontal radial wavenumber. Values of the integral $\int_{0}^{k}\left\langle\hat{w} \hat{\Theta}^{*}\right\rangle d k$ converge for $\Theta=b^{\prime}$, $g \alpha T^{\prime}, g \beta S^{\prime}$ with increasing wavenumber, suggesting the resolution is sufficient to capture the scalar fluxes. The cutoff frequency $k_{c}$ used in the $2 / 3$ dealiasing rule is included as a dashed vertical line.

explained by the fact that the salinity sublayer continues to grow on the diffusive time scale as the diffusive salt flux from the melting boundary is much larger than the turbulent vertical salt flux in the convecting region. On the other hand, the boundary heat flux rapidly comes into balance with the turbulent vertical heat flux (not shown). As the gravitationally stable haline sublayer grows, turbulence is damped out over a larger area close to the ice base, reducing the turbulent vertical heat flux. This leads to a reduction in the boundary heat flux, which coupled with the reduction in the boundary salt flux, reduces the melt rate on the time scale of the growing salinity sublayer. Eventually the boundary diffusive salt flux will come into balance with the turbulent vertical salt flux and the system can reach a steady state. In the cold, high mechanical forcing case $2 \mathrm{C}$, the system has almost reached this steady point near the end of the simulated period.

There is an imprint of convection on the spatial patterns of the instantaneous melt rate, although this effect is limited to cases in which $\left\langle w^{\prime} b^{\prime}\right\rangle$ is large compared to $\varepsilon_{0}$ (i.e., warm cases). Figure 7 shows snapshots of the melt rate for three simulations. In the cold case $2 \mathrm{C}$ the melt rate follows the patterns of the forced turbulence, illustrated by the passive case. In the warm, low mechanical forcing case $1 \mathrm{~B}$, where convection is strong, plume-like structures are visible in the melt rate. We may expect qualitatively different roughness patterns to develop on the underside of ice in the presence of strong double-diffusive

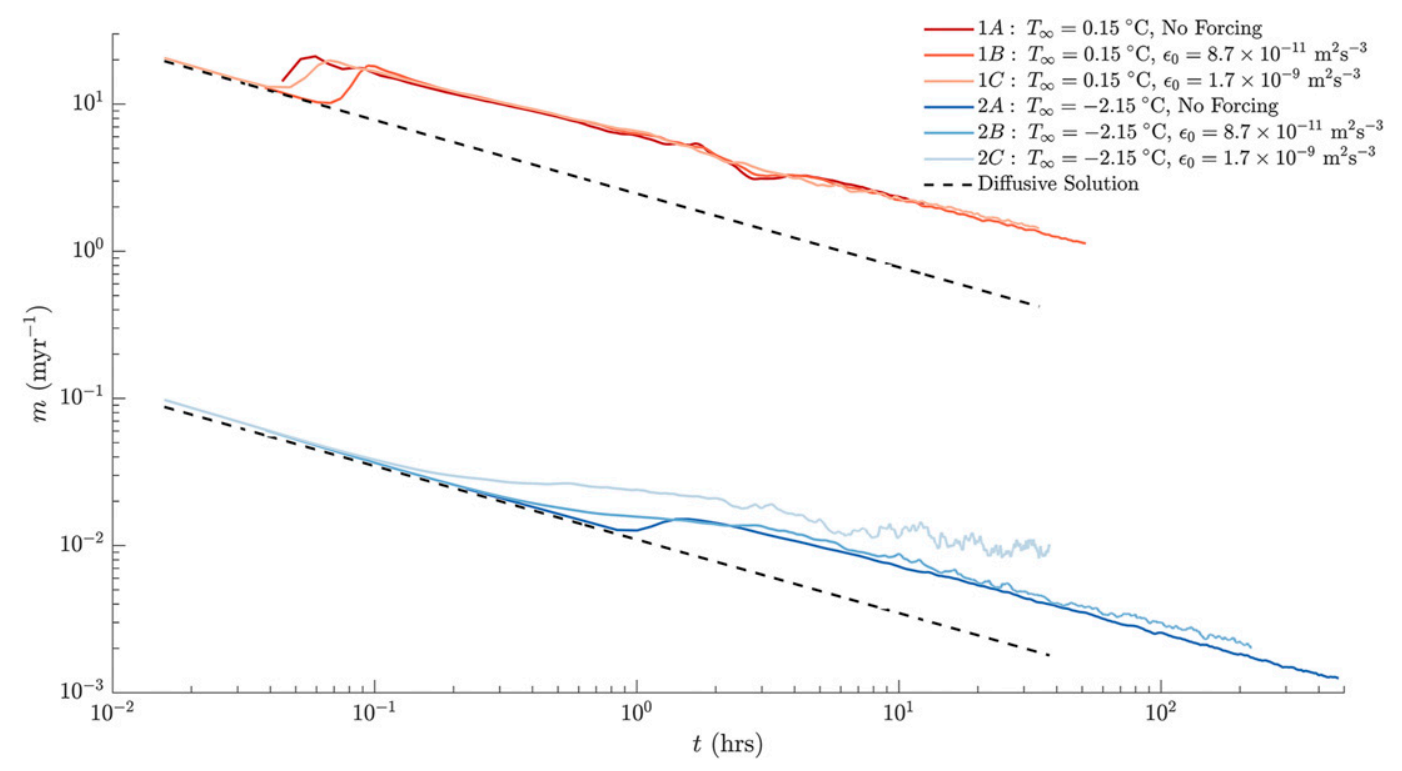

FIG. 6. Melt rate for cases 1A, 1B, 1C (the relatively warm cases) and for cases 2A, 2B, 2C (the relatively cold cases).

The diffusive solution (Martin and Kauffman 1977) is shown in a dotted line for both warm and cold cases. 

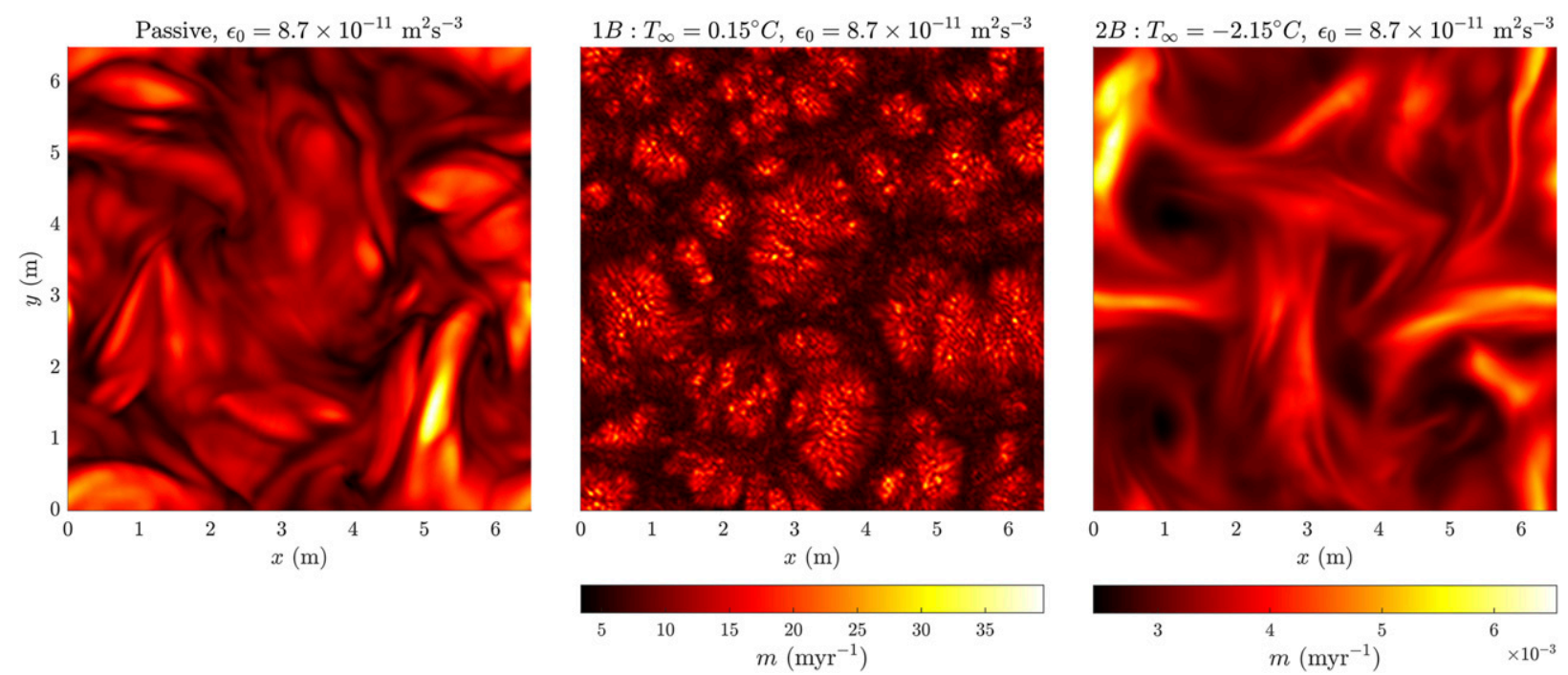

FIG. 7. Horizontal melt rate patterns for cases $1 B$ and 2B. Snapshots taken at the same time as in Fig. 4. Also included is a snapshot from the passive spinup simulation to compare patterning (melt rate values are inflated in this case due to lack of stable haline sublayer, so not included).

convection. However, including feedbacks from a moving boundary would be necessary to test this hypothesis.

\section{Diapycnal buoyancy flux}

In this section we identify a mechanism for the doublediffusive convection discussed within section 3 . We propose that the dominant forcing for double-diffusive convection is a region of negative diapycnal buoyancy flux near the ice base and we locate it based on a criterion given by Middleton and Taylor (2020). We first review the criterion and its motivation in terms of double-diffusive energetics.

\section{a. Background theory}

Here, we define the diapycnal buoyancy flux as the diffusive flux of buoyancy across surfaces of constant buoyancy, or isopycnals. For double-diffusive fluids, the diapycnal buoyancy flux can be upgradient, which corresponds to a negative buoyancy diffusivity (Radko 2013). The energetics of this was recently described by Middleton and Taylor (2020) as a diffusive release of "background" potential energy (BPE). Background potential energy is defined as the potential energy associated with an adiabatic rearrangement (i.e., sorting) of the density field, and "available" potential energy is the remaining potential energy after the background portion is subtracted. Winters et al. (1995) formalized the budget for the BPE for a single scalar, and showed the diapycnal buoyancy flux acts to transfer energy from APE to BPE, and so is associated with "irreversible mixing" (Winters et al. 1995). Extending the same framework, Middleton and Taylor (2020) showed that, in a double-diffusive fluid, the upgradient buoyancy flux corresponds to a conversion of BPE into APE which can then be modified into TKE via the turbulent vertical buoyancy flux $\left\langle w^{\prime} b^{\prime}\right\rangle$.

Middleton and Taylor (2020) provided a criterion for a negative diapycnal buoyancy flux in terms of the 3D scalar gradients. Specifically, the sign of the buoyancy flux is set by the following function,

$$
\operatorname{sgn}\left(\nabla b_{p} \cdot \hat{\mathbf{n}}\right)=\operatorname{sgn}\left[f\left(G_{\rho}, \theta, \frac{\kappa_{T}}{\kappa_{S}}\right)\right],
$$

where $\nabla b_{p}=g \alpha \kappa_{T} \nabla T-g \beta \kappa_{S} \nabla S$ is the diffusive buoyancy flux, $\hat{\mathbf{n}}=\nabla b /|\nabla b|$, and hence $\nabla b_{p} \cdot \hat{\mathbf{n}}$ is the diapycnal component of the diffusive buoyancy flux. The polynomial $f$ is

$$
f\left(G_{\rho}, \theta, \frac{\kappa_{T}}{\kappa_{S}}\right)=\frac{\kappa_{T}}{\kappa_{S}} G_{\rho}^{2}+\left(\frac{\kappa_{T}}{\kappa_{S}}+1\right) G_{\rho} \cos \theta+1,
$$

where the "gradient ratio", $G_{\rho}=\alpha|\nabla T| / \beta|\nabla S|$ is the $3 \mathrm{D}$ analog to the density ratio $R_{\rho}=\alpha(d T / d z) / \beta(d S / d z)$, and $\theta$ is the angle formed between the gradient vectors $\nabla S$ and $-\nabla T$. When $\theta=0$ the gradient vectors contribute to the buoyancy gradient constructively, and when $\theta=\pi$ they have opposing contributions to the buoyancy gradient. Negative values of $f$ (and an upgradient diapycnal buoyancy flux) require $\theta_{c}<\theta<2 \pi-\theta_{c}$, where

$$
\theta_{c}=\arccos \left(\frac{-2 \sqrt{\frac{\kappa_{T}}{\kappa_{S}}}}{\frac{\kappa_{T}}{\kappa_{S}}+1}\right) \sim 98.6^{\circ},
$$

where the $f<0$ region is bounded by $\theta_{c}$ and $2 \pi-\theta_{c}$. Generally, an upgradient diapycnal buoyancy flux is possible when the gradient vectors $\nabla T$ and $\nabla S$ make opposing contributions to the buoyancy gradient $\nabla b=g \alpha \nabla T-g \beta \nabla S$. The $f<0$ region is also bounded by $G_{\rho}=1$ and $G_{\rho}=\kappa_{T} / \kappa_{S}$, i.e., the salinity gradient must dominate the buoyancy gradient magnitude, but the temperature gradient must dominate the buoyancy flux gradient $\nabla b_{p}=g \alpha \kappa_{T} \nabla T-g \beta \kappa_{S} \nabla S$ magnitude. For $1 \mathrm{D}$ fields the angle $\theta=0$ or $\pi$, and restricting variation to the $z$ direction, this reduces to 

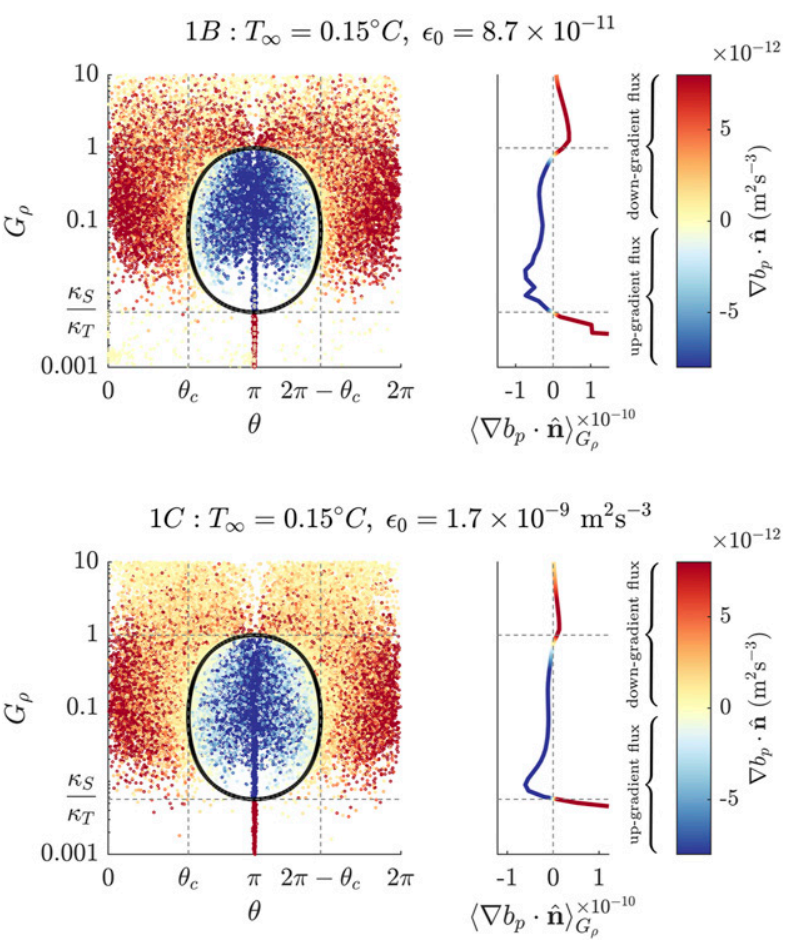
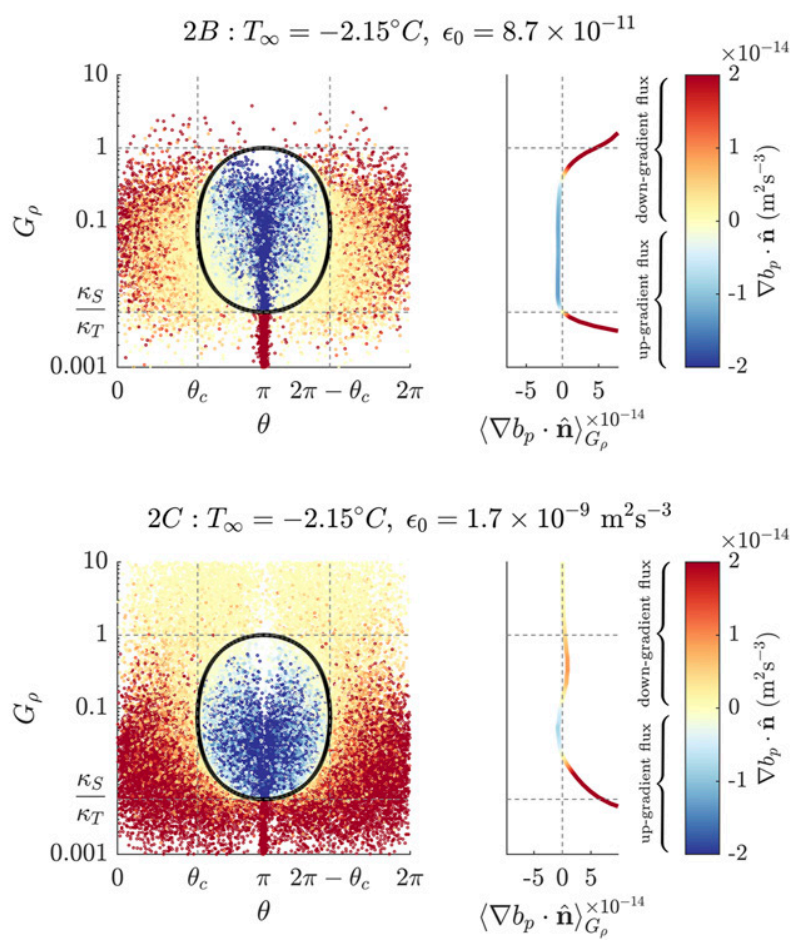

FIG. 8. Diapycnal buoyancy flux (color) for relatively warm cases $1 \mathrm{~B}$ and $1 \mathrm{C}$ and relatively cold cases $2 \mathrm{~B}$ and $2 \mathrm{C}$. 3D gradients are used to compute the diapycnal flux $\nabla b_{p} \cdot \hat{\mathbf{n}}$, the gradient ratio $G_{\rho}=\alpha|\nabla T| / \beta|\nabla S|$, and the angle $\theta$ between $-\nabla T$ and $\nabla S$. A random set of $1 / 1000$ of the points are then plotted as a scatter graph in $\left(G_{\rho}, \theta\right)$ space, colored by the diapycnal flux. The line $f\left(G_{\rho}, \theta\right)=0$ is plotted in black and divides the negative values of diapycnal flux (upgradient) on the inside of the line from the positive (downgradient) values outside of the line. To the right of each scatterplot is an average over the diapycnal flux across $G_{\rho}$ i.e., $\left\langle\nabla b_{p} \cdot \hat{\mathbf{n}}\right\rangle_{G_{\rho}}$, on the same color bar as the scatterplot. Note the gradient ratio $G_{\rho}$ is on a $\log$ scale.

$$
f<0 \Leftrightarrow \frac{\kappa_{S}}{\kappa_{T}}<R_{\rho}<1
$$

which is a well-known criterion for upgradient buoyancy flux in double diffusive fluids (Veronis 1965; St. Laurent and Schmitt 1999).

\section{b. Criterion for convection}

The cold, low mechanical forcing simulation $2 \mathrm{~B}$ shows a positive turbulent buoyancy flux (Fig. 3) despite a horizontally averaged density profile increasing with depth (Fig. 2) at late times, i.e., the turbulent buoyancy flux is upgradient. In this setting, convection is forced by preferential diffusion of temperature over salinity into fluid parcels near the ice/ocean boundary, causing increased density and forcing parcels to descend into the turbulent region below. In some cases this leads to a gravitationally unstable mean density profile. However, as case $2 \mathrm{~B}$ shows, convection can also occur when the mean density profile is stably stratified. The positive buoyancy flux in case $2 \mathrm{~B}$ is an example of an energy transfer from BPE to APE. Below, we examine this in detail by calculating the local diapycnal buoyancy flux.

To understand the influence of turbulence on the criterion for a negative diapycnal buoyancy flux, it is useful to consider the full 3D temperature and salinity fields. Figure 8 shows a scatterplot of the diapycnal buoyancy flux calculated from a
3D snapshot of the scalar fields for cases $1 \mathrm{~B}, 1 \mathrm{C}, 2 \mathrm{~B}$, and $2 \mathrm{C}$, in $\left(G_{\rho}, \theta\right)$ space. Here the criterion in Eq. (11) is exact and plotted as a black line. Also plotted is the diapycnal flux averaged for constant $G_{\rho}$. For $G_{\rho}<\kappa_{S} / \kappa_{T}$, the averaged diapycnal buoyancy flux $\left\langle\nabla b_{p} \cdot \hat{\mathbf{n}}\right\rangle_{G_{\rho}}$ is dominated by large positive values and $\theta \simeq \pi$. These points are located in the salinity sublayer, where $\nabla T$ and $\nabla S$ are nearly vertical. For other values of $G_{\rho}$, the points are spread across all angles $\theta$. This shows the role of turbulence in distorting temperature and salinity contours. In the nonconvecting case $2 \mathrm{C}$, there is scatter in $\theta$ even for $G_{\rho}<\kappa_{S} / \kappa_{T}$. In case $2 \mathrm{~B}$ convection is weak but active and $\left\langle w^{\prime} b^{\prime}\right\rangle$ is upgradient as the density profile is a monotonic function of height at the time shown. Here, the turbulent scatter is primarily restricted to the range $\kappa_{S} / \kappa_{T}<G_{\rho}<1$.

The points within the sublayer with $\theta \sim \pi$ are split into points with positive and negative diapycnal buoyancy flux. Close to the boundary $G_{\rho} \sim R_{\rho}<\kappa_{S} / \kappa_{T}$ and salinity forms the largest contribution to the buoyancy flux and buoyancy gradient. Farther from the boundary $G_{\rho} \sim R_{\rho}>\kappa_{S} / \kappa_{T}$ and temperature makes a larger contribution to the buoyancy flux while salinity still contributes most to the buoyancy gradient, leading to an upgradient buoyancy flux. In the convecting cases $1 \mathrm{~B}$ and $1 \mathrm{C}$, the $1 \mathrm{D}$ criterion $\kappa_{S} / \kappa_{T}<R_{\rho}<1$ for an upgradient buoyancy flux is sufficient to explain the averaged profile $\left\langle\nabla b_{p} \cdot \hat{\mathbf{n}}\right\rangle_{G_{\rho}}$ if we take $G_{\rho} \sim R_{\rho}$. However, in the marginally convecting case $2 \mathrm{~B}$, the $3 \mathrm{D}$ criterion is necessary to explain the 

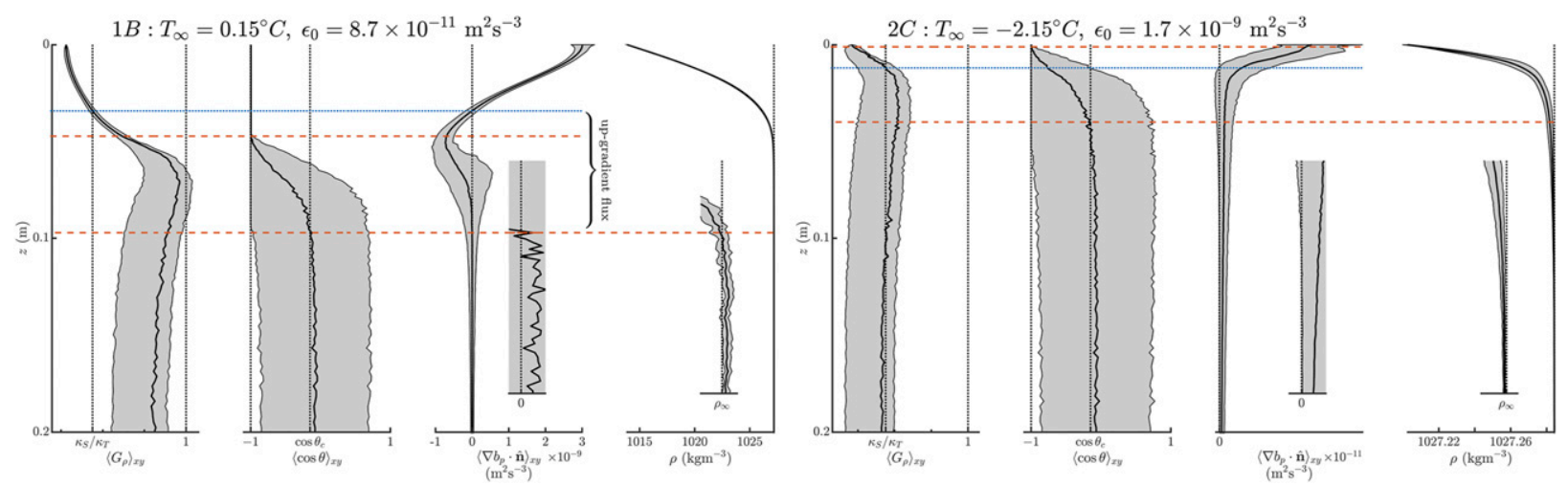

FIG. 9. Vertical profiles of the gradient ratio $G_{\rho}$, the scalar angle $\cos \theta$, the diapycnal flux $\nabla b_{p} \cdot \hat{\mathbf{n}}$, and the density $\rho$ in the upper $20 \mathrm{~cm}$ of a convecting simulation (warm, low mechanical forcing case $1 \mathrm{~B}$ ) and a nonconvective simulation (cold, high mechanical forcing case $2 \mathrm{C}$ ) at $t=30 \mathrm{~h}$. The spatial mean is shown in solid with one spatial standard deviation denoted by the shaded region. The dashed lines denote the depths at which $\langle\cos \theta\rangle_{x y}=-1$ and $\langle\cos \theta\rangle_{x y}=\cos \theta_{c}$. The dotted line denotes the depth at which $\left\langle G_{\rho}\right\rangle_{x y}=\kappa_{S} / \kappa_{T}$. The insets are a close-up version of the adjacent profiles on the same $z$ axis. The far-field density is denoted with a vertical dashed line in the plot of $\rho$. Note the gradient ratio $G_{\rho}$ in the left panel is on a log scale.

positive average diapycnal buoyancy flux for $0.2<G_{\rho}<1$ and in the nonconvecting case $2 \mathrm{C}$, the $1 \mathrm{D}$ approximation of $G_{\rho} \sim$ $R_{\rho}$ performs poorly.

Figure 9 shows profiles of horizontally averaged gradient ratio, scalar angle and diapycnal buoyancy flux from a convective simulation (case $1 \mathrm{~B}$ ) and a nonconvective simulation (case 2C) as a function of depth in the upper $20 \mathrm{~cm}$ at $t=30 \mathrm{~h}$. Shading indicates one standard deviation about the horizontal average. The depth where $\left\langle G_{\rho}\right\rangle=\kappa_{S} / \kappa_{T}$ is indicated with a blue dotted line, and the depths where $\langle\cos \theta\rangle=-1$ and $\langle\cos \theta\rangle=$ $\cos \theta_{c}$ are indicated with red dashed lines. At the ice base, the gradient ratio will always be less than $\kappa_{S} / \kappa_{T}$ as argued in section $2 b$. Farther from the ice, the temperature gradient exceeds the salinity gradient giving $\left\langle G_{\rho}\right\rangle=\kappa_{S} / \kappa_{T}$. The convective simulation shows significant negative diapycnal buoyancy flux in the region of $\left\langle G_{\rho}\right\rangle=\kappa_{S} / \kappa_{T}$ and $\langle\cos \theta\rangle<\cos \theta_{c}$ below the salinity sublayer and above the turbulent region. The critical angle $\theta_{c}$ is an exact bound on the local upgradient diapycnal buoyancy flux. However, it is not necessary that the horizontally averaged value gives the bound we see in the convecting case since the $3 \mathrm{D}$ criterion [Eq. (11)] is nonlinear. The negative diapycnal buoyancy flux peaks at the depth where $\langle\cos \theta\rangle=-1$ in all convecting simulations. Nonconvecting simulations have a positive mean diapycnal buoyancy flux at all depths, and the depth at which $\langle\cos \theta\rangle=-1$ is above the depth at which $\left\langle G_{\rho}\right\rangle=$ $\kappa_{S} / \kappa_{T}$. This implies that turbulence influences the distribution of angles $\theta$ in the region of $\left\langle G_{\rho}\right\rangle>\kappa_{S} / \kappa_{T}$ where otherwise it is possible to form an upgradient diapycnal buoyancy flux.

Figure 9 suggests that convection can be described using the relative thickness of two regions. The first is the region of $\left\langle G_{\rho}\right\rangle<\kappa_{S} / \kappa_{T}$, where the diapycnal buoyancy flux will always be downgradient. This region is determined to first order by the relative thickness of the temperature and salinity sublayers. The second is the region of $\langle\cos \theta\rangle=-1$, where turbulent velocities do not alter the temperature or salinity fields. When the second region is thicker than the first, there is a region where the scalar gradients $\nabla T$ and $\nabla S$ are vertical, and $\kappa_{S} / \kappa_{T}<R_{\rho}<1$, leading to an upgradient diapycnal buoyancy flux, which causes the release of BPE and subsequently double-diffusive convection. We can also identify the region of $\langle\cos \theta\rangle<\cos \theta_{c}$, where on average the angle between $\nabla T$ and $\nabla S$ is conducive to an upgradient diapycnal buoyancy flux. Below this region, $\cos \theta>\cos \theta_{c}$, and we expect the horizontally averaged diapycnal flux to be positive due to turbulent motions.

Parameters $G_{\rho}$ and $\theta$ are combinations of three-dimensional scalar gradients, and measuring these quantities in the field would be very challenging. It would be useful to have an approximate criterion that involves measurable quantities. In our simulations, the $G_{\rho}<\kappa_{S} / \kappa_{T}$ region is well described by the 1D approximation $R_{\rho}<\kappa_{S} / \kappa_{T}$. Our simulations also show a strong monotonic relationship, in a statistically averaged sense, between the angle $\theta$ and a common metric for turbulence, the buoyancy Reynolds number,

$$
\operatorname{Re}_{b}=\frac{\varepsilon}{\nu N^{2}}
$$

where $N^{2}=\partial b / \partial z$ is the buoyancy frequency. We calculate $\operatorname{Re}_{b}$ using horizontally averaged values for both $\varepsilon$ and $N^{2}$. The buoyancy Reynolds number quantifies the extent of the inertial subrange of the energy spectrum i.e., the separation between the Kolmogorov scale (the scale below which viscous effects dominate) and the Ozmidov scale (the scale above which buoyancy effects dominate). The buoyancy Reynolds number has also been used to identify double diffusion in the open ocean (Inoue et al. 2007).

For $\mathrm{Re}_{b}<1$ the flow will be laminar (Smyth and Moum 2000), and hence we might expect $\cos \theta \simeq-1$. The region of $\cos \theta<\cos \theta_{c}$ is well described by $\operatorname{Re}_{b}<10$, so for simulations with a region of negative mean diapycnal buoyancy flux, this region is bounded by $\mathrm{Re}_{b}=10$. For $1<\mathrm{Re}_{b}<10$ we can consider the flow very weakly turbulent, and for larger buoyancy Reynolds numbers $\operatorname{Re}_{b}>10$, we find the turbulence is sufficiently developed to give $\langle\cos \theta\rangle>\cos \theta_{c}$, causing a positive mean diapycnal buoyancy flux. 

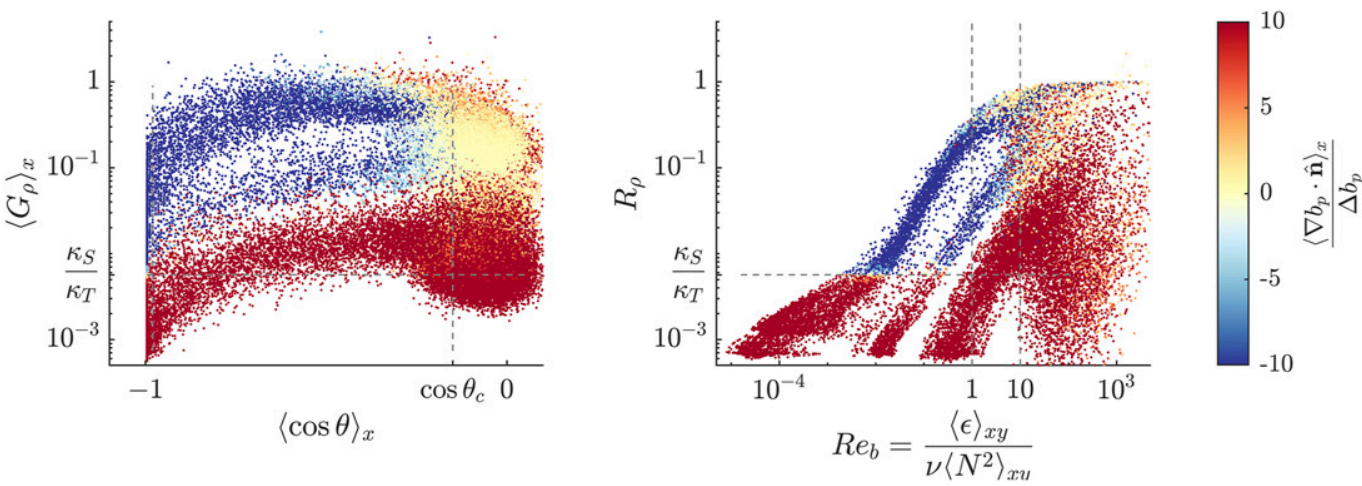

FIG. 10. Diapycnal buoyancy flux (color) for cases $1-6$ in Table 1 plotted (left) in $\left(G_{\rho}, \theta\right)$ space and (right) in $\left(R_{\rho}\right.$, $\mathrm{Re}_{b}$ ) space. The diapycnal buoyancy flux is normalized by the maximum (i.e., initial) difference $\Delta b_{p}=b_{p}^{\text {bottom }}-b_{p}^{\text {top }}$ across each simulation for comparison. The points were sampled from $2 \mathrm{D} x-z$ slices extracted from the 3D simulations at regular intervals.

Figure 10 shows how the horizontal mean diapycnal buoyancy flux varies with $G_{\rho}, \theta$, and $\mathrm{Re}_{b}$. Each point was calculated from $2 \mathrm{D}$ slices of the scalar fields at regular intervals throughout the simulations. The $2 \mathrm{D}$ slices are taken throughout the simulated period for all of the simulations conducted (listed in Table 1), and the mean profiles from all the sampled times are plotted in Fig. 10. For simulations with a density peak there are values of $\mathrm{Re}_{b}<0$; however, the region of interest is adjacent to the ice base so only points above the density peak are included. The points are plotted in $\left(G_{\rho}, \theta\right)$ space, where the coloration denotes the magnitude of the diapycnal buoyancy flux. The left panel shows that the region with an upgradient buoyancy flux (blue points) is mostly bounded by $\left\langle G_{\rho}\right\rangle>\kappa_{S} / \kappa_{T}$ and $\cos \theta<$ $\cos \theta_{c}$, indicated using dashed lines. For the nonconvecting simulations, there are points with positive diapycnal flux for $\langle\cos \theta\rangle<\cos \theta_{c}$ and $G_{\rho}>\kappa_{S} / \kappa_{T}$, which does not occur for the convecting simulations.

The right panel in Fig. 10 plots the same data as the left panel, but now as a function of $R_{\rho}$ and $\mathrm{Re}_{b}$. The gradient ratio $G_{\rho}$ is a good approximation to the density ratio $R_{\rho}$ in the diffusive sublayer, but they differ in the turbulent region. In all simulations, $\operatorname{Re}_{b}<1$ adjacent to the ice, suggesting that the near-ice region is laminar. If turbulent eddies existed close to the ice they would feel the effect of the wall, but no such eddies occur due to the strength of the stratification. The points that lie within the region $R_{\rho}>\kappa_{S} / \kappa_{T}$ and $\mathrm{Re}_{b}<1$ have a negative diapycnal buoyancy flux and these points occur at the top of the boundary layer in the convecting simulations. This leads to the following hypothesis: Convection will occur at the melting ice base if the depth at which $\operatorname{Re}_{b}=1$ is deeper than the depth at which $R_{\rho}=\kappa_{S} / \kappa_{T}$. In the next section we will use this criterion to extend our results to a wider range of parameters.

\section{Discussion}

The criterion for diffusive convection described in section $4 b$ allows us to extrapolate our results to a wider range of parameters. For example, given a temperature and salinity profile, we can find the dissipation rate $\varepsilon$ required to give $\mathrm{Re}_{b}=1$ at the depth where $R_{\rho}=\kappa_{S} / \kappa_{T}$. In practice, field measurements of $T / S$ profiles within the ice shelf-ocean boundary layer cannot yet resolve the diffusive sublayers, with reliable measurements limited to depths of $\mathscr{O}(10) \mathrm{cm}$. However, observations may be combined with assumptions and models to estimate the relative depths of $\mathrm{Re}_{b}=1$ and $R_{\rho}=\kappa_{S} / \kappa_{T}$. This provides an estimate for the dissipation rate above which turbulence suppresses diffusive convection.

In the absence of $T / S$ profiles, we can estimate the conditions that will be favorable for diffusive convection by considering the development of diffusive boundary layers into a fluid with initially uniform temperature and salinity. The solution of the unsteady diffusion equations forced by the melt boundary condition was derived by Martin and Kauffman (1977). Since heat diffuses down faster than salt, the density profile will be initially unstable with the potential to trigger diffusive convection. Over time, the addition of freshwater from melting will deepen the haline sublayer, decreasing the salinity gradient, and so decreasing $N^{2}$ in the halocline at the depth where $R_{\rho}=\kappa_{S} / \kappa_{T}$. The reduction in $N^{2}$ increases $\operatorname{Re}_{b}$. Once $\operatorname{Re}_{b}>1$ at the depth where $R_{\rho}=\kappa_{S} / \kappa_{T}$, turbulence begins to suppress the upgradient buoyancy flux which maintains diffusive convection.

Given an initial temperature, salinity and dissipation rate, we can calculate the time taken for the diffusive solution to meet our criterion for the shutdown of convection. This time is shown in Fig. 11 for a fixed salinity $S_{\infty}=34.572$, matching our simulations, while varying the initial temperature, $T_{\infty}$ (normalized by the freezing temperature $T_{m}=\lambda_{1} S_{\infty}+\lambda_{2}+\lambda_{3} P$ ), and the rate of dissipation $\varepsilon$. Note that the diffusive solution does not account for turbulent mixing of the temperature and salinity profiles, so the transition times will not be quantitatively accurate. However, the diffusive solution provides a point of comparison between different levels of thermal forcing and rates of dissipation.

The simulations listed in Table 1 are included in Fig. 11 for comparison. Convecting simulations are marked with circles and nonconvecting simulations are marked with crosses and can be separated using a transition time of $t=1 \mathrm{~s}$. This indicates that the predicted transition time might be a useful way to distinguish between convecting and nonconvecting states in terms of their bulk parameters. The parameter space suggested 


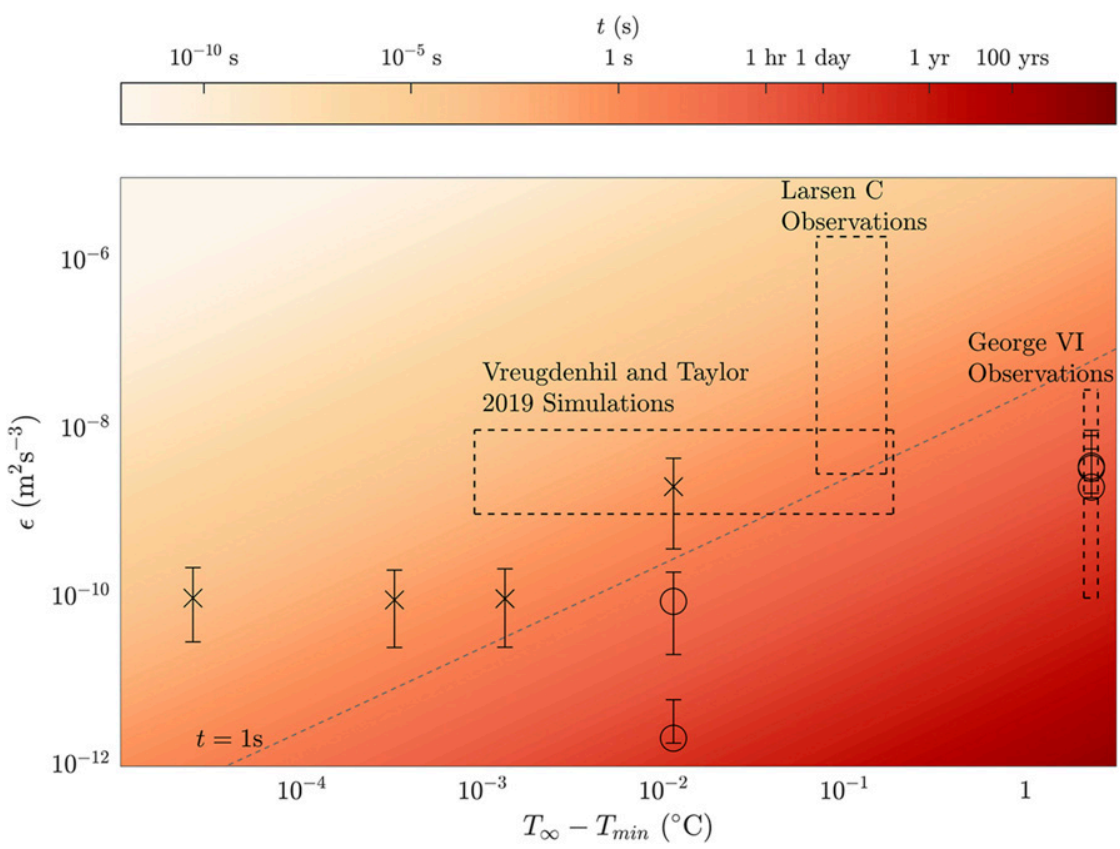

FIG. 11. Predicted time required for the system to transition from diffusive convection to stratified turbulence, calculated with the diffusive solution (Martin and Kauffman 1977) with far field temperature $T_{\infty}$ and prescribed turbulent dissipation rate $\varepsilon$. "Transition" occurs when $\operatorname{Re}_{b}=1$ at $R_{\rho}=\kappa_{S} / \kappa_{T}$. The far-field salinity $S_{\infty}=34.572 \mathrm{ppt}$ in all cases and $T_{\min }=\lambda_{1} S_{\infty}+\lambda_{2}+$ $\lambda_{3} P$ is the freezing temperature. Simulation values of $\varepsilon_{\text {measured }}$ are given by markers with bounds indicating maximum and minimum values. Circular markers indicate convecting simulations and cross markers indicate nonconvecting simulations. The contour for diffusive solutions to take $t=1 \mathrm{~s}$ to transition is marked as a dividing point between the convecting and nonconvecting simulations. Regions of parameter space occupied by the observations from Larsen C Ice Shelf (Davis and Nicholls 2019), George VI Ice Shelf (Venables et al. 2014), and the LES for a shear driven boundary layer (Vreugdenhil and Taylor 2019) shown with dashed boxes.

by the observations of Larsen C Ice Shelf (Davis and Nicholls 2019) and George VI Ice Shelf (Venables et al. 2014) as well as the parameter space explored in the shear-driven large-eddy simulations of Vreugdenhil and Taylor (2019) (inferred from a law-of-the-wall scaling) are marked using dashed boxes. The parameter space for the Larsen C Ice Shelf observations and the LES cover relatively short transition times, indicating double-diffusive convection may not occur. However, the George VI Ice Shelf parameter space has a long transition time and hence the flow is amenable to transient double-diffusive convection as suggested by Kimura et al. (2015).

In the simulations and analysis here, we used idealized initial conditions with uniform temperature and salinity. However, diffusive convection can occur in other configurations. For example, consider a turbulent ice-ocean boundary layer in a nonconvecting steady state. If turbulence levels decrease (e.g., due to weakening currents), the buoyancy Reynolds number will decrease, so the depth at which $\mathrm{Re}_{b}=1$ will increase. If this depth at which $\mathrm{Re}_{b}=1$ becomes deeper that the depth at which $R_{\rho}=\kappa_{S} / \kappa_{T}$ then the criterion from section $4 \mathrm{~b}$ is satisfied and convection will ensue. The double-diffusive convection preferentially transports heat over salt, so we would expect the salt boundary layer to grow slowly. Therefore a boundary layer in warm or weakly turbulent conditions may take a long time to adjust to modest changes in turbulence levels.

\section{Conclusions}

Motivated by observations made beneath George VI ice shelf in Antarctica, we conducted a series of numerical simulations of an idealized ocean boundary layer beneath a melting ice shelf. The simulations were initialized with constant salinity and temperature and the evolution of the system under a thickening salt sublayer was studied.

Two distinct flow regimes were observed. In one regime, the mean density profile increased with depth and the density field acted to damp the forced turbulence. This is the standard assumption in stratified melting ice-ocean boundary parameterizations. In the other regime, doublediffusive convection occurred and potential energy was converted into kinetic energy, forced by an upgradient buoyancy flux in a region near the ice base. All simulations started in the convective regime, but some quickly transitioned to a turbulence-damping state. Simulations in different regimes exhibit qualitatively different patterns in the velocity field, melt rate and melt pattern. 
We examined the influence of temperature and ambient turbulence levels on the flow regime by systematically varying the initial and far-field temperature and the strength of the mechanical forcing. A criterion for an upgradient buoyancy flux and hence double-diffusive convection using the 3D scalar gradients (Middleton and Taylor 2020) was applied to the simulation data. This criterion identified the region of upgradient diapycnal buoyancy flux near the ice base, responsible for convection.

We developed a simple prediction for an upgradient buoyancy flux (Middleton and Taylor 2020) at the ice base based on local values of the density ratio and the buoyancy Reynolds number $\mathrm{Re}_{b}$. We found double-diffusive convection if the depth of the region beneath the ice of $R e_{b} \lesssim 1$ is deeper than the region of $R_{\rho} \lesssim \kappa_{S} / \kappa_{T}$. We then used solutions from the unsteady diffusion equations from Martin and Kauffman (1977) to estimate when the boundary layer will be favorable to double-diffusive convection based on the turbulent dissipation rate and the far field temperature.

The interaction of melt-driven convection with thermohaline layering and anisotropic turbulence (including shear) could modify some of our conclusions, and in particular the specific value of the buoyancy Reynolds number used in the criterion for the shutdown of double-diffusive convection could be sensitive to the source of turbulence. This could be investigated in future studies. We anticipate that the principles used here to distinguish between externally forced turbulence and double-diffusive convection could be applied to other settings and will be a useful starting point in future work.

Our results indicate that melt-driven double-diffusive convection can dominate the dynamics within the ice shelfocean boundary layer if the turbulence is sufficiently weak and/or the thermal driving is sufficiently large. This study suggests future ice-ocean boundary layer parameterizations may need to distinguish between convective and nonconvective conditions in the melting regime. However, more observations in warm, weakly turbulent conditions are needed to assess the prevalence of double-diffusive convection beneath ice shelves.

Acknowledgments. This work was funded by grants from the Natural Environment Research Council, NE/N009746/1 and NE/N010027/1.

\section{APPENDIX}

\section{Grid Stretching}

The Kolmogorov length scale gives a measure of the smallest turbulent eddies and is defined as

$$
\eta=\left(\frac{\nu^{3}}{\varepsilon}\right)^{1 / 4}
$$

We resolve the Kolmogorov scale within the "observation region" of our domain $(z<2.6 \mathrm{~m})$. The diffusivities for heat and salt are smaller than the diffusivity for momentum

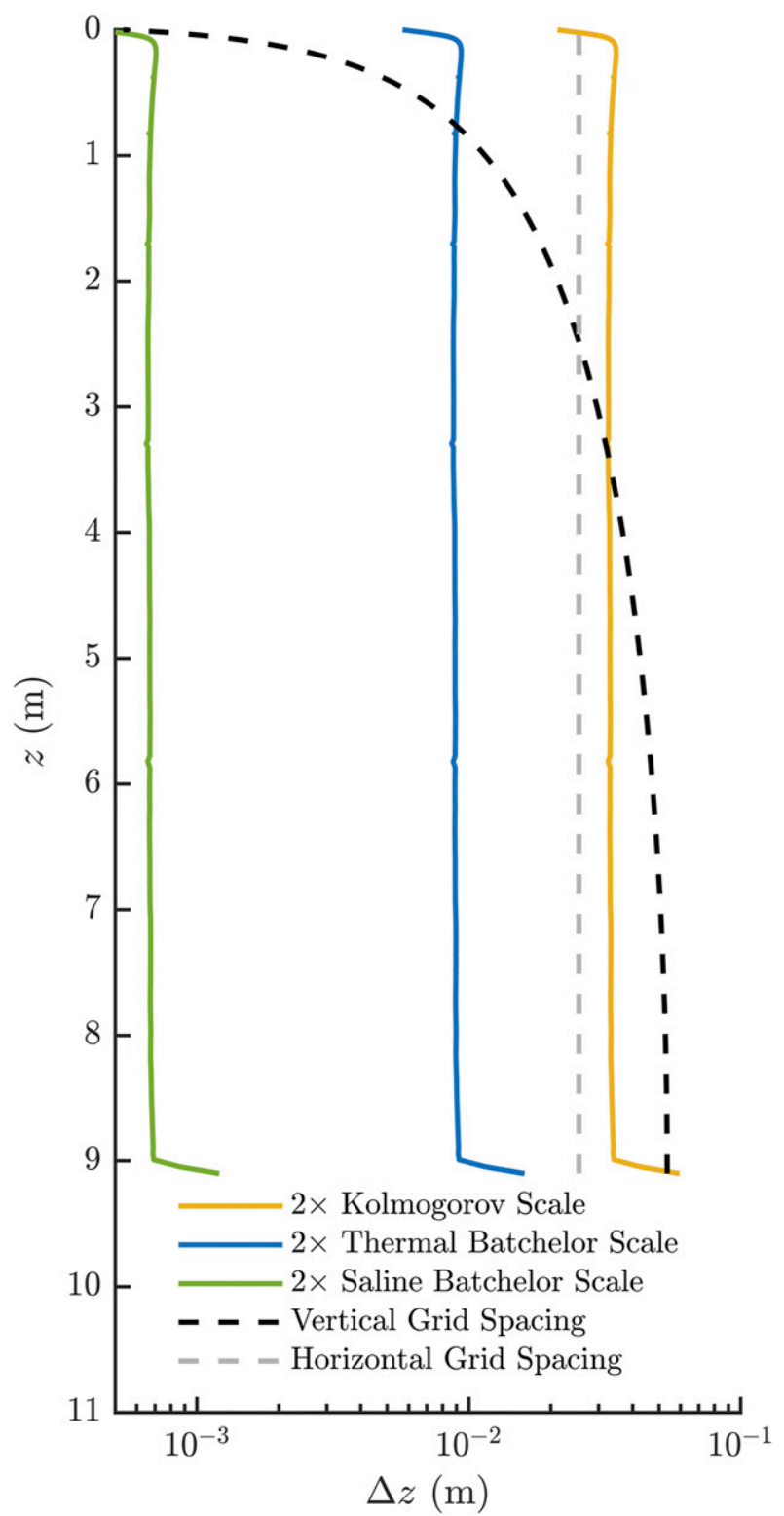

FIG. A1. Grid spacing plotted with depth. Kolmogorov and Batchelor scales for both scalars are shown, with dissipation rates taken from passive simulation for $\varepsilon_{0}=8.7 \times 10^{-11} \mathrm{~m}^{2} \mathrm{~s}^{-3}$.

(i.e., kinematic viscosity $\nu$ ), so variability on scales smaller than the Kolmogorov scale is possible. These scales are quantified using the thermal and haline Batchelor scales, defined as

$$
l_{B}^{T}=\frac{\eta}{\operatorname{Pr}^{1 / 2}}=\left(\frac{\nu \kappa_{T}^{2}}{\varepsilon}\right)^{1 / 4}, \quad l_{B}^{S}=\frac{\eta}{\mathrm{Sc}^{1 / 2}}=\left(\frac{\nu \kappa_{S}^{2}}{\varepsilon}\right)^{1 / 4} .
$$

Figure A1 shows the grid spacing graphically, comparing the distance between grid points $\Delta z$ to the Kolmogorov and Batchelor scales, calculated based on the dissipation rate profile of the passive spin up. We have compared our grid spacing to the turbulent length scales multiplied by a factor of 2 as a commonly argued factor. 
Vreugdenhil and Taylor (2018) found that including seven grid points within the conducting sublayer was sufficient to resolve the diffusive fluxes at the wall in their simulations of stratified plane Couette flow, a criterion which was then applied to resolve salt fluxes in the ice-ocean boundary layer simulations of Vreugdenhil and Taylor (2019). We use the same criterion here, and we have further verified that our simulations follow the analytical diffusive solution (see Fig. 2) at early times, before turbulent mixing increases the haline sublayer thickness. Using the definition of the salinity sublayer as when the salinity reaches $99 \%$ of its far field value, we find that, for the diffusive solution, we have seven grid points within the haline sublayer after $t=25 \mathrm{~min}$. Before this time, the simulated scalar fields match the diffusive solution which suggests the scalar fluxes are resolved at the boundary throughout our simulations.

\section{REFERENCES}

Begeman, C. B., and Coauthors, 2018: Ocean stratification and low melt rates at the Ross Ice Shelf grounding zone. J. Geophys. Res. Oceans, 123, 7438-7452, https://doi.org/10.1029/2018JC013987.

Carpenter, J. R., T. Sommer, and A. Wüest, 2012: Simulations of a double-diffusive interface in the diffusive convection regime. J. Fluid Mech., 711, 411-436, https://doi.org/10.1017/ jfm.2012.399.

Crapper, P., 1976: Fluxes of heat and salt across a diffusive interface in the presence of grid generated turbulence. Int. J. Heat Mass Transf., 19, 1371-1378, https://doi.org/10.1016/ 0017-9310(76)90065-X.

Davis, P. E., and K. W. Nicholls, 2019: Turbulence observations beneath Larsen C ice shelf, Antarctica. J. Geophys. Res. Oceans, 124, 5529-5550, https://doi.org/10.1029/2019JC015164.

Frank, F. C., 1950: Radially symmetric phase growth controlled by diffusion. Proc. Roy. Soc., 201A, 586-599, https://doi.org/ 10.1098/rspa.1950.0080.

Gade, H. G., 1979: Melting of ice in sea water: A primitive model with application to the Antarctic ice shelf and icebergs. J. Phys. Oceanogr., 9, 189-198, https://doi.org/10.1175/ 1520-0485(1979)009<0189:MOIISW > 2.0.CO;2.

Gayen, B., R. W. Griffiths, and R. C. Kerr, 2016: Simulation of convection at a vertical ice face dissolving into saline water. J. Fluid Mech., 798, 284-298, https://doi.org/10.1017/jfm.2016.315.

Guthrie, J. D., J. H. Morison, and I. Fer, 2013: Revisiting internal waves and mixing in the Arctic Ocean. J. Geophys. Res. Oceans, 118, 3966-3977, https://doi.org/10.1002/jgrc.20294.

Hickel, S., N. Adams, and N. Mansour, 2007: Implicit subgrid-scale modeling for large-eddy simulation of passive-scalar mixing. Phys. Fluids, 19, 095102, https://doi.org/10.1063/1.2770522.

Holland, D. M., and A. Jenkins, 1999: Modeling thermodynamic iceocean interactions at the base of an ice shelf. J. Phys. Oceanogr., 29, 1787-1800, https://doi.org/10.1175/1520-0485(1999)029<1787: MTIOIA $>2.0 . \mathrm{CO} ; 2$.

Holland, P. R., A. Jenkins, and D. M. Holland, 2010: Ice and ocean processes in the Bellingshausen Sea, Antarctica. J. Geophys. Res., 115, C05020, https://doi.org/10.1029/2008JC005219.

Inoue, R., H. Yamazaki, F. Wolk, T. Kono, and J. Yoshida, 2007: An estimation of buoyancy flux for a mixture of turbulence and double diffusion. J. Phys. Oceanogr., 37, 611-624, https:// doi.org/10.1175/JPO2996.1.

Jenkins, A., K. W. Nicholls, and H. F. Corr, 2010: Observation and parameterization of ablation at the base of Ronne Ice Shelf,
Antarctica. J. Phys. Oceanogr., 40, 2298-2312, https://doi.org/ 10.1175/2010JPO4317.1.

Keitzl, T., J. P. Mellado, and D. Notz, 2016: Reconciling estimates of the ratio of heat and salt fluxes at the ice-ocean interface. J. Geophys. Res. Oceans, 121, 8419-8433, https://doi.org/ 10.1002/2016JC012018.

Kimura, S., K. W. Nicholls, and E. Venables, 2015: Estimation of ice shelf melt rate in the presence of a thermohaline staircase. J. Phys. Oceanogr., 45, 133-148, https://doi.org/10.1175/ JPO-D-14-0106.1.

Martin, S., and P. Kauffman, 1977: An experimental and theoretical study of the turbulent and laminar convection generated under a horizontal ice sheet floating on warm salty water. J. Phys. Oceanogr., 7, 272-283, https://doi.org/ 10.1175/1520-0485(1977)007<0272:AEATSO>2.0.CO;2.

McPhee, M. G., G. A. Maykut, and J. H. Morison, 1987: Dynamics and thermodynamics of the ice/upper ocean system in the marginal ice zone of the Greenland Sea. J. Geophys. Res., 92, 7017-7031, https://doi.org/10.1029/JC092iC07p07017.

Merryfield, W. J., 2000: Origin of thermohaline staircases. J. Phys. Oceanogr., 30, 1046-1068, https://doi.org/10.1175/ 1520-0485(2000)030<1046:OOTS $>2.0$. CO 2 .

Middleton, L., and J. Taylor, 2020: A general criterion for the release of background potential energy through double diffusion. J. Fluid Mech., 893, R3, https://doi.org/10.1017/ jfm.2020.259.

Orszag, S. A., 1971: On the elimination of aliasing in finite-difference schemes by filtering high-wavenumber components. J. Atmos. Sci., 28, 1074, https://doi.org/10.1175/1520-0469(1971)028<1074: OTEOAI $>2.0 . \mathrm{CO} ; 2$.

Phillips, O., 1972: Turbulence in a strongly stratified fluid-Is it unstable? Deep-Sea Res. Oceanogr. Abstr., 19, 79-81, https:// doi.org/10.1016/0011-7471(72)90074-5.

Radko, T., 2013: Double-Diffusive Convection. Cambridge University Press, 344 pp.

Rao, K., and S. de Bruyn Kops, 2011: A mathematical framework for forcing turbulence applied to horizontally homogeneous stratified flow. Phys. Fluids, 23, 065110, https://doi.org/ 10.1063/1.3599704.

Rignot, E., S. Jacobs, J. Mouginot, and B. Scheuchl, 2013: Ice-shelf melting around Antarctica. Science, 341, 266-270, https:// doi.org/10.1126/science.1235798.

Scalo, C., U. Piomelli, and L. Boegman, 2012: Large-eddy simulation of oxygen transfer to organic sediment beds. J. Geophys. Res., 117, 6005, https://doi.org/10.1029/2011JC007289.

Shibley, N., and M.-L. Timmermans, 2019: The formation of double-diffusive layers in a weakly turbulent environment. J. Geophys. Res. Oceans, 124, 1445-1458, https://doi.org/ 10.1029/2018JC014625.

Smyth, W. D., and J. N. Moum, 2000: Length scales of turbulence in stably stratified mixing layers. Phys. Fluids, 12, 1327-1342, https://doi.org/10.1063/1.870385.

St. Laurent, L., and R. Schmitt, 1999: The contribution of salt fingers to vertical mixing in the North Atlantic Tracer Release Experiment. J. Phys. Oceanogr., 29, 1404-1424, https://doi.org/10.1175/1520-0485(1999)029<1404:TCOSFT $>$ 2.0.CO;2.

Taylor, J. R., 2008: Numerical simulations of the stratified oceanic bottom boundary layer. Ph.D. thesis, University of California, San Diego, 212 pp.

— turbulent water. Science, 338, 675-679, https://doi.org/10.1126/ science.1219417. 
, S. M. de Bruyn Kops, C. P. Caulfield, and P. F. Linden, 2019: Testing the assumptions underlying ocean mixing methodologies using direct numerical simulations. J. Phys. Oceanogr., 49, 2761-2779, https://doi.org/10.1175/JPO-D-19-0033.1.

Turner, J., 1968: The behaviour of a stable salinity gradient heated from below. J. Fluid Mech., 33, 183-200, https://doi.org/ 10.1017/S0022112068002442.

Venables, E., K. Nicholls, F. Wolk, K. Makinson, and P. Anker, 2014: Measuring turbulent dissipation rates beneath an Antarctic ice shelf. Mar. Technol. Soc. J., 48, 18-24, https:// doi.org/10.4031/MTSJ.48.5.8.

Veronis, G., 1965: On finite amplitude instability in thermohaline convection. J. Mar. Res., 23 (1), 1-17.

Vreugdenhil, C. A., and J. R. Taylor, 2018: Large-eddy simulations of stratified plane Couette flow using the anisotropic minimum-dissipation model. Phys. Fluids, 30, 085104, https://doi.org/10.1063/1.5037039.

- and —, 2019: Stratification effects in the turbulent boundary layer beneath a melting ice shelf: Insights from resolved large-eddy simulations. J. Phys. Oceanogr., 49, 1905-1925, https://doi.org/10.1175/JPO-D-18-0252.1.

Wang, L.-P., S. Chen, J. G. Brasseur, and J. C. Wyngaard, 1996: Examination of hypotheses in the Kolmogorov refined turbulence theory through high-resolution simulations. Part I: Velocity field. J. Fluid Mech., 309, 113-156, https://doi.org/ 10.1017/S0022112096001589.

Winters, K. B., P. N. Lombard, J. J. Riley, and E. A. D'Asaro, 1995: Available potential energy and mixing in density-stratified fluids. J. Fluid Mech., 289, 115-128, https://doi.org/10.1017/ S002211209500125X. 


\section{University Library}

\section{- M M N E R VA A gateway to Melbourne's research publications}

Minerva Access is the Institutional Repository of The University of Melbourne

Author/s:

Middleton, L;Vreugdenhil, CA;Holland, PR;Taylor, JR

Title:

Numerical Simulations of Melt-Driven Double-Diffusive Fluxes in a Turbulent Boundary Layer beneath an Ice Shelf

Date:

2021-02

\section{Citation:}

Middleton, L., Vreugdenhil, C. A., Holland, P. R. \& Taylor, J. R. (2021). Numerical Simulations of Melt-Driven Double-Diffusive Fluxes in a Turbulent Boundary Layer beneath an Ice Shelf. Journal of Physical Oceanography, 51 (2), pp.403-418. https://doi.org/10.1175/jpod-20-0114.1.

Persistent Link:

http://hdl.handle.net/11343/268107 